Linköping University Medical Dissertation No. 1738

\title{
Colorectal Liver Metastases
}

- Different Aspects on Treatment with Associated Liver Partition and Portal Vein Ligation for Staged hepatectomy and on Portal Vein Occlusion

Kristina Hasselgren 


\title{
Colorectal Liver Metastases - Different Aspects on Treatment
} with Associated Liver Partition and Portal Vein Ligation for Staged Hepatectomy and on Portal Vein Occlusion

\author{
Kristina Hasselgren \\ Division of Surgery \\ Department of Biomedical and Clinical Sciences \\ Linköping University \\ Linköping 2020
}




\section{Copyright @ Kristina Hasselgren, 2020}

Kristina.Hasselgren@liu.se

Published articles have been reprinted with the permission of the copyright holder.

Printed in Sweden by LiU-Tryck. Linköping, Sweden, 2020

ISSN 0345-0082

ISBN 978-91-7929-860-9 


\section{Supervisor}

Bergthor Björnsson, MD, PhD, Associate Professor

Division of Surgery

Department of Biomedical and Clinical Sciences

Linköping University

\section{Assistant supervisor}

Per Sandström, MD, PhD, Professor

Division of Surgery

Department of Biomedical and Clinical Sciences

Linköping University

\section{Former assistant supervisor}

Tommy Sundqvist, PhD, Professor emeritus

Division of Medical Microbiology

Department of Biomedical and Clinical Sciences

Linköping University

\section{Opponent}

Per Lindner, MD, PhD, Associate Professor

Department of Transplantation and Liver Surgery, Sahlgrenska Academy

University of Gothenburg

\section{Host}

Bergthor Björnsson, MD, PhD, Associate Professor

Division of Surgery

Department of Biomedical and Clinical Sciences

Linköping University 


\section{Committee board}

Preben Kjölhede, MD, PhD, Professor (chairman)

Division of Obstetrics and Gynecology

Department of Biomedical and Clinical Sciences

Linköping University

Torsten Olbers, MD, PhD, Professor

Division of Surgery

Department of Biomedical and Clinical Sciences

Linköping University

Peter Matthiessen MD, PhD, Associate Professor

Division of Surgery

Department of Health and Medicine

Örebro University

Pär Myrelid MD, PhD, Professor

Division of Surgery

Department of Biomedical and Clinical Sciences

Linköping University

Anders Kald MD, PhD, Associate Professor

Division of Surgery

Department of Biomedical and Clinical Sciences

Linköping University 
"det dunkelt sagda är det dunkelt tänkta"

Esaias Tegner, Epilog vid Magister-promotionen i Lund 1820 



\section{Contents}

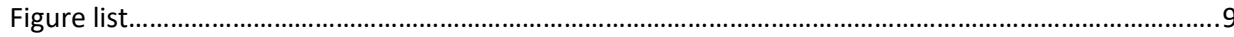

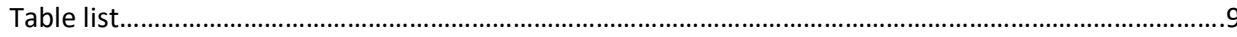

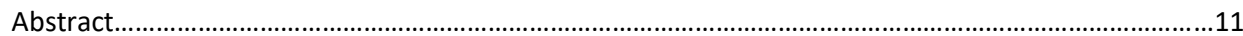

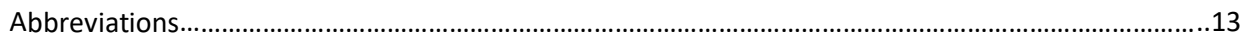

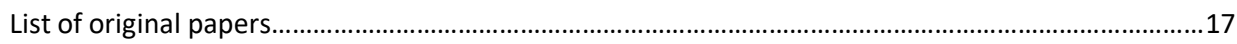

1. Colorectal liver metastases....................................................................................................19

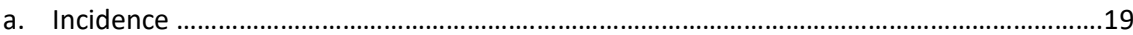

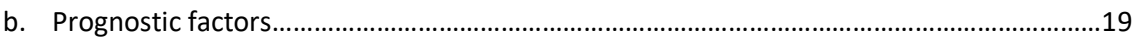

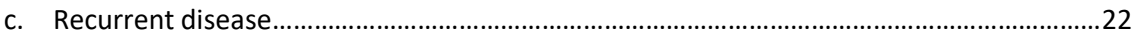

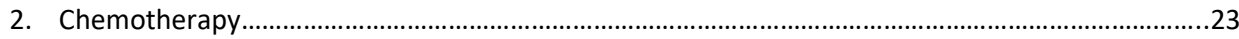

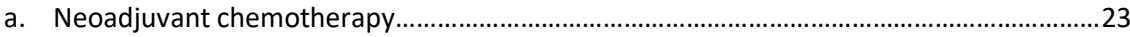

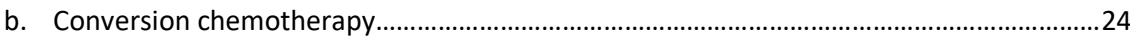

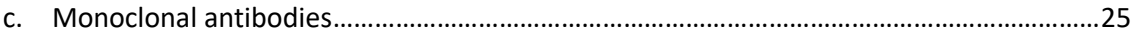

d. Chemotherapy and postoperative complications................................................................25

e. Chemotherapy and its impact on the volume increase of the future liver remnant.........26

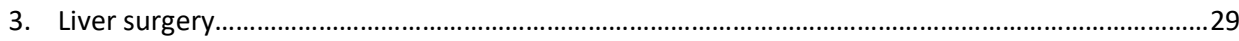

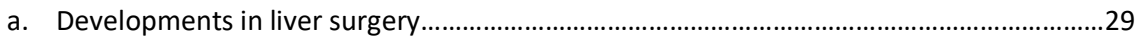

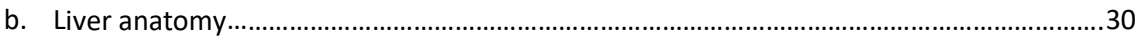

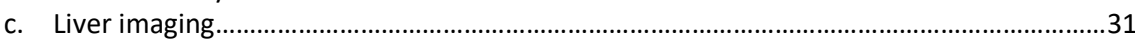

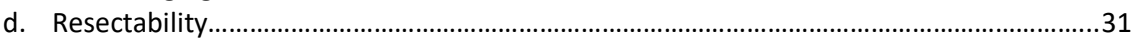

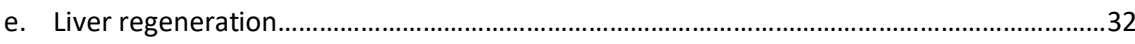

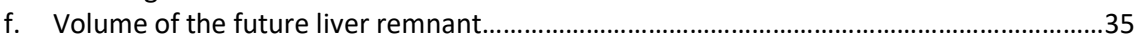

g. Techniques to increase the volume of the future liver remnant..........................................37

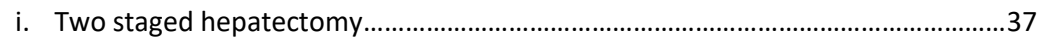

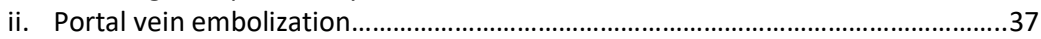

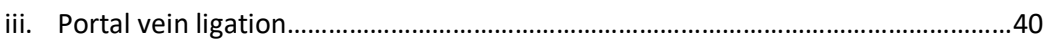

iv. Concerns regarding portal vein occlusion.................................................................41

v. Associating liver partition and portal vein ligation for staged hepatectomy.........41

vi. Other techniques to achieve radical resection for patients with advanced

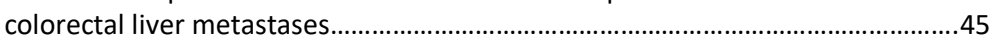

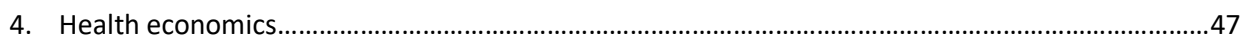

a. Health economic evaluation of patients with colorectal liver metastases..........................48

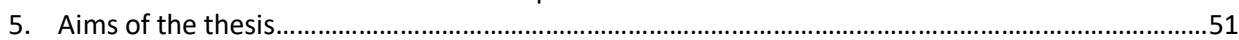

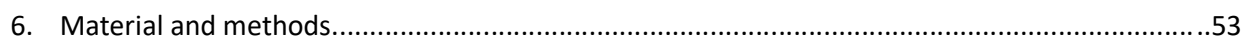

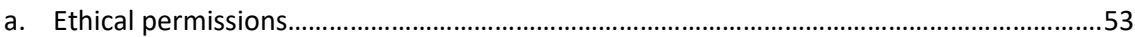

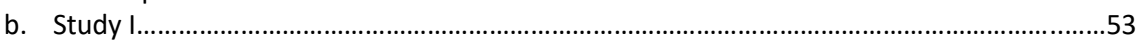

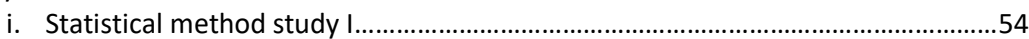

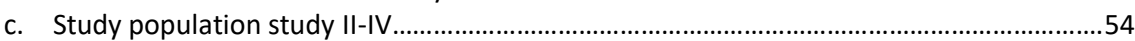

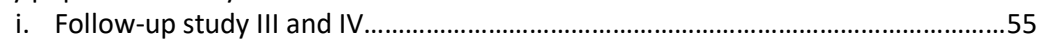

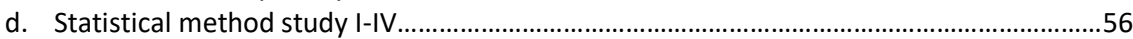

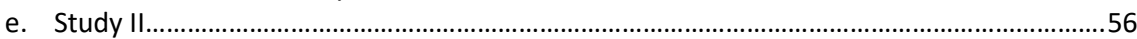

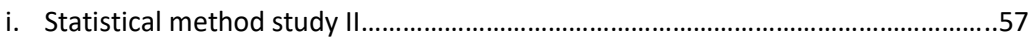

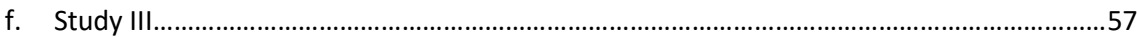

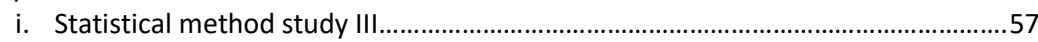

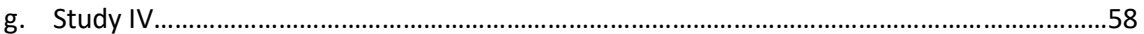




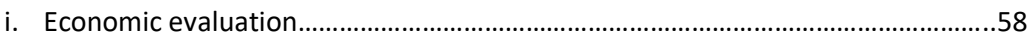

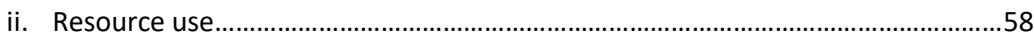

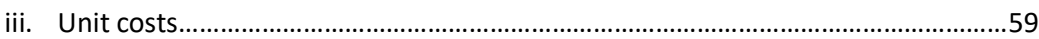

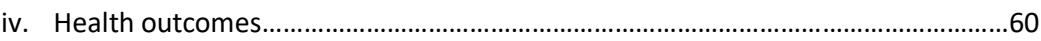

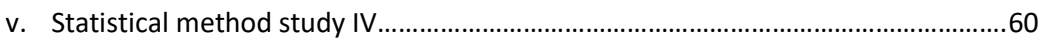

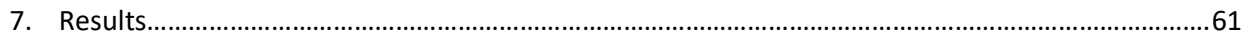

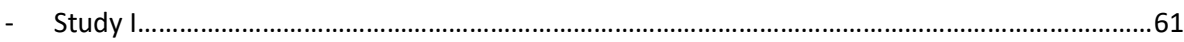

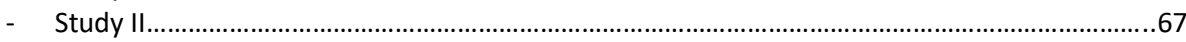

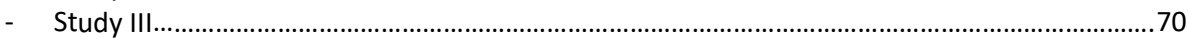

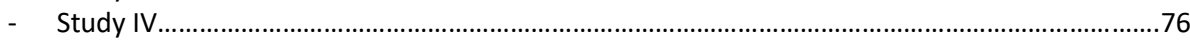

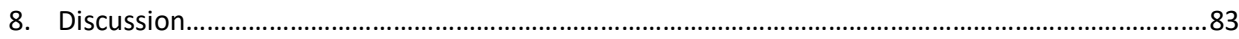

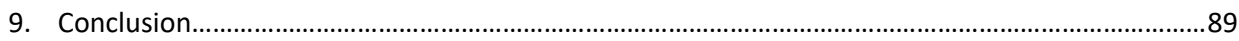

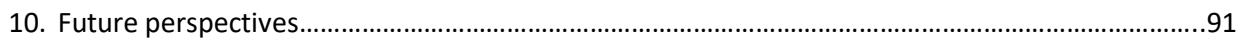

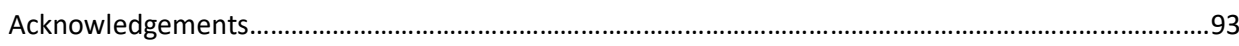

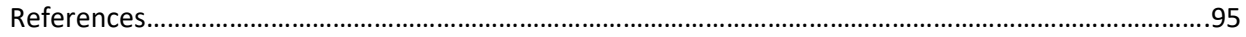




\section{Figure list}

Figure 1. Volume increase of the future liver remnant in percent (study I)

Figure 2. Volume increase of the future liver remnant in milliliter (study I) 65

Figure 3. Volume increase of the future liver remnant as kinetic growth rate (study I). .66

Figure 4. Temporal course of the volume increase of the future liver remnant (study II). .68

Figure 5. Estimated median survival for patients resected with ALPPS, TSH, rescue ALPPS and those not resected in both groups (study III).

Figure 6. Incremental cost and QALY for ALPPS compared to TSH (study IV)..... 81

Figure 7. Incremental cost and LY for ALPPS compared to TSH (study IV). .82

\section{Table list}

Table 1. Clinical and preoperative volumes (study I)..... 61

Table 2. Description of preoperative chemotherapy (study I) .63

Table 3. Clinical variables and preoperative data (study II). 67

Table 4. Clinical and preoperative data (study III and study IV).

Table 5. Uni- and multivariable Cox regression analysis (study III)... .75

Table 6. Included costs (study IV). .77 


\section{Abstract}

Introduction: For patients with colorectal liver metastases (CRLM), the only treatment with a possibility for long-term survival and cure is radical resection. The majority of patients are at the time of diagnosis not assessed as resectable because they have advanced disease in the liver or unresectable extrahepatic disease or are too frail to withstand liver surgery.

Patients who at the time of diagnosis are not assessed as resectable may be treated with conversion chemotherapy to downsize the tumor burden and render the patient eligible for resection. One concern with chemotherapy administered preoperatively has been the potential negative effect on the future liver remnant (FLR), especially for patients with a low volume of the FLR who are undergoing techniques to increase the volume. Established techniques to increase the volume are portal vein occlusion (PVO) and two-staged hepatectomy (TSH). A more recent method is Associating Liver Partition and Portal Vein Ligation for Staged Hepatectomy (ALPPS). Due to the relative novelty of ALPPS, the long-term oncological results are not known.

For patients with CRLM, resection of liver metastases is more favorable from a health economic perspective than palliative treatment and results in a higher quality of life than palliative chemotherapy. For patients undergoing ALPPS as well as TSH, the data are scarce.

Aim: The aim of the first study was to determine whether preoperative chemotherapy has a negative impact on the volume increase for patients undergoing ALPPS. The aim of the second study was to analyze the temporal course of the volume increase in the FLR for patients undergoing PVO. The aim of the third study was to study the long-term outcome for patients randomized to ALPPS or TSH. The aim of the fourth study was to perform a health economic analysis of patients randomized to ALPPS or TSH.

Methods: The first study was based on data from the ALPPS registry, which is an international registry initiated 2012. All patients included in the registry between 2012 and 2016 were included. 
The patients were divided into the following four groups: no preoperative chemotherapy, 1 regimen of neoadjuvant chemotherapy, more than 1 regimen, and more than 1 regimen with the addition of monoclonal antibodies. The volume increase between interventions 1 and 2 was analyzed. In the second study, a retrospective analysis was performed of patients randomized to TSH. Forty-eight patients were included. The volume increase of the FLR was analyzed as the kinetic growth rate (KGR). The KGR was calculated from PVO until radical hepatectomy or exclusion, as well as between the first and second radiological evaluations. In the third and fourth studies, patients randomized to ALPPS and TSH were included. In the third study, survival, as well as factors affecting the outcome, were analyzed. In the fourth study, a calculation of resource use was performed, as was an analysis of health-related quality of life (HRQoL) for the groups.

Results: In the first study, it was found that chemotherapy had no negative impact on the volume increase for patients undergoing ALPPS. In the second study, it was found that the volume increase of the FLR was largest the first week after ALPPS. In the third study, it was found that patients randomized to ALPPS had a longer survival than those randomized to TSH. Of the factors affecting the outcome, resection of liver metastases had a significant impact. In the fourth study, no significant difference could be found in resource use or HRQoL for patients randomized to ALPPS over TSH.

Conclusion: Patients with advanced CRLM undergoing ALPPS should receive preoperative chemotherapy, if indicated. For those undergoing PVO, early evaluation is crucial to evaluate the volume increase, and for those with insufficient increase, additional techniques to increase the volume should be considered. Resection of liver metastases is an important factor to improve the outcome. Further studies are warranted to conclude whether ALPPS or TSH is most effective from a health economic perspective. 


\section{Abbreviations}

ALPSS - associating liver partition and portal vein ligation in staged hepatectomy

ALTPS - associating liver tourniquet and portal ligation for staged hepatectomy

BSA - body surface area

CALI - chemotherapy-associated liver injury

CEA - carcinoembryonic antigen

CRLM - colorectal liver metastases

CT - computed tomography

CUSA - cavitron ultrasonic aspirator

DFS - disease-free survival

EGF - epidermal growth factor

EHD- extrahepatic disease

e-OSH - enhanced one-stage ultrasound-guided hepatectomy

$\mathrm{EVOH}$ - ethylene vinyl alcohol

FLR - future liver remnant

HCC - hepatocellular carcinoma

HGF - hepatocyte growth factor

IL-6 - interleukin 6

INR - international normalized ratio

ISGLS - International Study Group of Liver Surgery 
K-RAS - Kirsten rat sarcoma viral oncogene

LVD - liver venous deprivation

$\mathrm{MRI}$ - magnetic resonance imaging

NBCA - N-butyl-cyanoacrylate

OS - overall survival

PET - positron emission tomography

PHLF - posthepatectomy liver failure

PVA - polyvinyl alcohol

PVE - portal vein embolization

PVL - portal vein ligation

PVO - portal vein occlusion

RCT - randomized controlled trial

RFA - radiofrequency ablation

RO - microscopic radical resection

R1 - microscopic residual disease

SD - sinusoidal dilatation

SFLR - standardized future liver remnant

TBS - tumor burden score

TGF- $\beta$ - transforming growth factor beta

TNF - tumor necrosis factor 
TLV - total liver volume

TSH - two-staged hepatectomy

US - ultrasound 
List of original papers

K. Hasselgren, M. Malago, S. Vyas, R. Robles Campos, R. Brusadin, M. Linecker, H. Petrowsky, P. A. Clavien M. Autran Machado, R. Hernandez-Alejandro, K. Wanis, L. Valter, P. Sandström, P. Sandström, B. Björnsson.

Neoadjuvant chemotherapy does not affect future liver remnant growth and outcomes of associating liver partition and portal vein ligation for staged hepatectomy Surgery 2017;16(5)1255-1265

K. Hasselgren, P. Sandström, BI. Røsok, E. Sparrelid, G. Lindell, P. Nørgaard Larsen, A. Lindhoff Larsson, NA. Schultz, BA. Bjørnbeth, B. Isaksson, M. Rizell, B. Björnsson.

Future liver remnant (FLR) increase in patients with colorectal liver metastases is highest the first week after portal vein occlusion

Journal of Gastrointestinal Surgery 2019;23(3)556-562

K. Hasselgren, BI. Røsok, PN. Larsen, E. Sparrelid, G. Lindell, NA. Schultz, BA. Bjørnbeth, B. Isaksson, A. Lindhoff Larsson, M. Rizell, B. Björnsson, P. Sandström.

ALPPS Improves Survival Compared with TSH in Patients Affected of CRLM

Annals of Surgery 2019. Online ahead of print

K. Hasselgren, M. Henriksson, BI. Røsok, PN. Larsen, E. Sparrelid, G. Lindell, NA. Schultz, BA.

Bjørnbeth, B. Isaksson, M. Rizell, A. Lindhoff Larsson, P. Sandström, B. Björnsson

Health economic evaluation of patients with colorectal liver metastases randomized to ALPPS or TSH - analysis from the LIGRO trial, manuscript 


\section{Colorectal liver metastases}

Incidence

Colorectal cancer is one of the most common malignant diseases in the Western world, with approximately 1.8 million new cases each year [1]. Fifteen to twenty percent of patients with colorectal cancer present with synchronous (within 6 months from the diagnosis of the primary tumor) liver metastases [2, 3]. Approximately $30 \%$ of patients who have undergone resection of the primary tumor will develop metachronous liver metastases up to 10 years from the diagnosis of the primary tumor [4].

\section{Prognostic scoring systems and prognostic factors}

Patients with colorectal liver metastases (CRLM) are diverse, and hence, their prognoses differ. The most crucial factor is whether resection is possible. Although no randomized controlled trial has been performed regarding resection, resection is stated as the only treatment with the possibility for cure and long-term survival.

The need for scoring systems is due to the high recurrence rate, which is reported to be up to nearly $50 \%$ [5]; the scoring systems aim to predict which patients will benefit from liver resection and which patients may not benefit.

For patients treated with resection, there are several scoring systems. The scoring systems compile various factors, and it is thus possible to estimate the prognosis. One of the first scoring systems was developed by Nordlinger et al [6]. It is based on data from 85 centers and on patients resected between the late 1960s and the 1990s. It consists of 7 factors that were used to create three risk groups [6]. Another of the earliest scoring systems was the Fong score. The Fong score was developed in the 1990s and is based on patients who underwent resection during the latter half of the 1980s and 1990s. Seven factors were found to be significantly associated with prognosis. 
Radicality of the resection, occurrence of EHD (extrahepatic disease), nodal status of the primary tumor, disease-free interval less than 12 months, number of liver metastases, largest CRLM $>5 \mathrm{~cm}$ and CEA (carcinoembryonic antigen) $>200 \mathrm{ng} / \mathrm{l}$. Of those, nodal status, disease-free interval, number of liver metastases, size of the largest liver metastasis and CEA were combined into a clinical risk score [7]. One advantage of the Fong score is that preoperative known clinical factors are included. An objection against such scoring systems is that they were developed before modern chemotherapy $[8,9]$.

Since then, a wide range of prognostic factors and scoring systems have been investigated. They can broadly be divided into those employing clinical factors and those employing molecular factors.

The location of the primary tumor appears to have prognostic significance, and right-sided primary tumors have a worse prognosis than left-sided primary tumors [10, 11]. Regarding the impact of time of diagnosis of CRLM in relation to the primary tumor on prognosis, data indicate that synchronous CRLM has a worse prognosis than metachronous CRLM, while other studies could not demonstrate an impact on the prognosis $[12,13]$. Furthermore, data indicate a difference in molecular markers for synchronous compared to metachronous CRLM, which would indicate a difference in tumor biology [14].

The number of liver metastases appears to have prognostic significance, although there is no clear cut-off value [15, 16], as does the size of the metastases [17]. A relatively new scoring system, named the tumor burden score (TBS), in which the number of tumors as well as the maximum tumor size is incorporated into one value has been proposed [18].

The radicality of liver resection is of prognostic significance, and patients who undergo R1 resection (microscopic residual disease) have a worse outcome than those who undergo R0 resection [19-21]. The results regarding the impact of the width of the tumor-free margin on survival are conflicting, and it has been shown that a $1 \mathrm{~mm}$ margin results in comparable long-term survival to a wider resection margin [22] and that a tumor-free margin of more than $1 \mathrm{~mm}$ is associated with improved 
outcome [21]. Factors associated with R1 resection are factors associated with more advanced tumor burden in the liver, such as long operating time, more than tree tumors, larger metastases and bilobar metastases [23].

CEA is a widely accepted and clinically used tumor marker. In the 1970s, elevated CEA was used to predict recurrence of colorectal cancer, among recurrence of other tumors [24]. CEA is recognized as a prognostic marker, although the cut-off value remains to be established [19, 25].

In terms of molecular factors and oncogenes, KRAS (Kirsten rat sarcoma viral oncogene) is one of the most studied and one of the most frequently mutated oncogenes in CRLM. A mutation in KRAS is associated with shorter overall survival (OS) and disease free survival (DFS) [26-28]. KRAS has also been incorporated into a clinical risk score [29].

Two fundamentally different growth patterns are seen on histopathological examination and are described as desmoplastic and pushing. Of those, the pushing growth pattern is associated with inferior outcomes [30].

Furthermore, data indicate that the same factors cannot be used for predicting the prognosis for patients with recurrent disease in the liver [31], and there is a difference in the weight of prognostic factors for patients with synchronous compared to metachronous tumors [32].

It has also been recognized that the postoperative course impacts the outcome. Postoperative infectious complications have been found to be associated with decreased long-term survival. Risk factors for infectious complications include major liver resections [33].

The research field is rapidly expanding, although there is currently no answer to which factors determine the outcome. There is a consensus that for patients for whom radical resection is possible, liver resection should be considered. 


\section{Recurrent disease}

Up to nearly $50 \%$ of patients previously treated with liver resection will develop recurrent disease in the liver during follow-up [5]. Approximately $70 \%$ of patients with recurrent disease limited to the liver are eligible for curative intent resection or ablation. For those treated with resection, survival is comparable with survival after primary hepatectomy [34].

For patients with advanced CRLM, who require a two-stage approach to achieve radical resection, the recurrence rate is higher; approximately $77 \%$ develop recurrent disease during follow-up. This category of patients has bilobar CRLM, and upfront resection would result in a too low of a volume of the future liver remnant (FLR). A lower proportion (53\%) of patients are treated with repeat resection, and the outcome for those patients is more favorable than that for those not treated with repeat resection [35].

Risk factors for recurrence are a more advanced T stage of the primary tumor, synchronous CRLM, more than 3 CRLMs and bilobar metastases [16]. 


\section{Chemotherapy}

Progress in oncological therapy has rapidly increased in recent decades. It was nearly 50 years ago that one of the first descriptions of the use of a combination of multiple cytotoxic agents, including fluorouracil, was published [36]. Irinotecan was developed in the 1990s and was initially used as a second-line treatment for patients with stage IV colorectal cancer for which treatment with fluorouracil failed [37]. Oxaliplatin was introduced in the 1990s for patients with metastatic colorectal cancer [38].

Today, chemotherapy has become important in the multimodal treatment of patients with CRLM. Chemotherapy may be given preoperatively, either for neoadjuvant purposes or with the intent to convert the metastases to resectable. To be assessed as resectable, the future liver remnant must be of sufficient volume, eventual extrahepatic disease must be resectable, and the patient's performance status and eventual comorbidities must allow for liver resection to be performed with an acceptable risk of postoperative complications [39].

Chemotherapy may also be given postoperatively for adjuvant purposes. It may also be administered to patients who are assessed as requiring palliative care.

\section{Neoadjuvant chemotherapy}

It is not completely clear whether all patients benefit from preoperative chemotherapy. Patients who are assessed as eligible for resection may not benefit from neoadjuvant chemotherapy [40], although it may result in longer progression-free survival [41, 42]. For patients with single metachronous metastases, no difference in survival has been shown when compared to those resected up front [43]. For patients with more advanced disease, although assessed as eligible for resection, they have been shown to have an improved survival if treated with neoadjuvant chemotherapy compared to those with a similar tumor burden who undergo upfront resection [44]. 
In summary, no firm evidence exists regarding whether patients who are technically assessed as eligible for resection benefit from neoadjuvant chemotherapy.

\section{Conversion chemotherapy}

Conversion chemotherapy refers to chemotherapy administered to patients with unresectable liver metastases with the purpose of attaining resectability [45].

Approximately 13 to $40 \%$ of patients who are assessed as ineligible for resection at presentation may become eligible for resection after conversion chemotherapy [39, 46]. The five-year survival ranges from 33 to $47 \%$ in resected patients $[39,46]$. Some studies indicate that the survival for patients who undergo resection after conversion chemotherapy is comparable with the survival for those who undergo up front resection [47-50]. However, the DFS is shorter in the conversion group [48-50].

The groups are, however, disparate; in one study, no patients had EHD, and no patients were treated with PVO (portal venous occlusion) prior liver resection $[49,50]$, compared to another study in which a fifth of the patients were treated for PVO and approximately $15 \%$ had irresectable EHD at diagnosis, before the start of conversion chemotherapy [48]. In another study, no patient with EHD at diagnosis became eligible for resection [47]. 


\section{Monoclonal antibodies}

Monoclonal antibodies have been used as therapeutic agents since the 1980s and are used in a wide array of different diseases. The monoclonal antibodies used in CRLM are mainly bevacizumab, cetuximab and panitumumab, and they were developed in the beginning of the 2000s [51].

Bevacizumab is a monoclonal antibody targeting vascular endothelial growth factor (VEGF). VEGF is a key factor in the development of blood vessels, including tumor blood vessels. By inhibiting VEGF, the formation of new blood vessels in tumors is impeded, and it is also believed that existing blood vessels may regress [52]. For patients with advanced CRLM who are initially assessed as ineligible for resection, the addition of bevacizumab to chemotherapy increases the resectability rate and therefore the proportion of patients potentially eligible for treatment with curative intent [53].

Cetuximab and panitumumab are monoclonal antibodies targeting epidermal growth factor receptor. EGFR is essential for cell proliferation, and activation of the receptor initiates the process. Cetuximab and panitumumab are indicated in those with no mutation in KRAS (wild-type) [54]. In patients with mutations in KRAS, proliferation is not dependent on EGFR activation [55]. For patients with wild-type KRAS who are ineligible for resection at diagnosis, the addition of cetuximab or panitumumab increases the resection rate $[56,57]$.

\section{Chemotherapy and postoperative complications}

There is a general concern that preoperative chemotherapy is associated with an increased risk of postoperative complications. Whether the risk is increased for all patients treated with preoperative chemotherapy and regardless of the extent of liver resection is not fully established. There are data indicating that the risk is not increased for patients treated with preoperative chemotherapy [58]. However, the majority of patients in that study did not undergo major liver resection. 
The risk of posthepatectomy liver failure (PHLF) is increased for patients treated with preoperative chemotherapy [59], especially patients undergoing major liver resection and with a low volume of the FLR (future liver remnant) if treated with long-term preoperative chemotherapy [60]. FLR is the remaining part of the liver after liver resection. The addition of monoclonal antibodies does not seem to increase the risk of postoperative complications, provided a sufficient interval, in general about 6 weeks, between the last dose and liver resection exists [61, 62].

Preoperative chemotherapy may also induce histological alterations in the liver and even chemotherapy-associated liver injury (CALI). CALI can further be divided into sinusoidal obstruction syndrome, which is associated with oxaliplatin [63], and steatohepatitis, which is associated with irinotecan [64]. Severe SD (sinusoidal dilatation) is associated with an increased risk of severe postoperative complications, and steatohepatitis increases the risk of liver-specific postoperative complications [65].

\section{Chemotherapy and its impact on increased future liver remnant volume}

Patients with an estimated low volume of the FLR are often treated with preoperative chemotherapy since they often have advanced disease. Whether oncological therapy, including monoclonal antibodies, has a negative impact on the volume increase for patients treated with techniques to increase the volume of the FLR has been contested.

Previous studies show conflicting results and are furthermore based on a relatively small number of patients, making it difficult to draw a firm conclusion. In some studies, oncological therapy, including bevacizumab, resulted in a smaller increase in the volume of the FLR after PVO, especially for older patients and for patients treated with more than 6 cycles of bevacizumab [66]. These results were confirmed by another study, in which longer chemotherapy resulted in a lower increase of the FLR. These results are, however, slightly difficult to generalize since nearly $50 \%$ of the patients were re- 
embolized, indicating that a part of the insufficient volume increase was due to suboptimal PVE [67]. Other studies show no difference in volume increase when comparing patients treated with chemotherapy lasting until a few weeks before hepatectomy, with interruption a few weeks before and after PVO, to those who ended the treatment 1 month before PVO [68]. However, it cannot be denied that an eventual negative impact of chemotherapy may exist more than a month prior to completion of treatment. In another study, no difference in volume increase could be found. However, the chemotherapy group had a significantly higher frequency of liver steatosis, indicating that the impact of chemotherapy remained but not to such an extent that a difference in volume increase could be found [69]. 


\section{Liver Surgery}

\section{Developments in liver surgery}

One of the first liver resections in a nontrauma setting was performed in 1888 by the German surgeon Carl Johann August Langenbuch [70]. One of the first descriptions of major liver surgery for a malignant indication was published in 1899. What appears to be a left hemihepatectomy was performed, and the patient was described to recover from the surgery, although some of the surgical techniques and postoperative measurements would not be accepted today. Other techniques, albeit further developed, remain today, such as the use of a heat source (diathermy) and the application of pressure to the liver to control bleeding [71]. During the first half of the twentieth century, there were only sporadic publications regarding liver surgery. During the 1950s, the segmental liver anatomy was described, mainly by Claude Couinaud, which is fundamental to modern liver surgery [72].

With the development of radiological imaging techniques, starting with ultrasound and computed tomography during the 1980s, it became possible to detect small lesions [73]. Intraoperative ultrasound is a further milestone in liver surgery [74]. During the 1980s, liver resection based on liver anatomy resulted in further improvements [75].

Many techniques for transection of the liver parenchyma have been described, including the finger fracture technique, which was subsequently developed into Kelly's technique (the clamp crush technique), ultrasonic dissection (of which CUSA (cavitron ultrasonic aspirator) was the first), the Hydrojet dissector and the radiofrequency dissection sealer [76, 77]. Larger blood vessels, especially the hepatic veins, are generally divided with staples [78]. There is no evidence that any method is superior in terms of postoperative morbidity or in terms of reducing perioperative blood loss. 
Control of the inflow to the liver and with temporal occlusion (Pringle maneuver) can further reduce bleeding [79]. Another important advance to reduce blood loss during liver resection is the application of controlled, low venous pressure during liver surgery [80].

\section{Liver anatomy}

A fundamental understanding of the liver anatomy is crucial for the liver surgeon, both in preoperative planning and during liver resection. A challenge in truly comprehending the liver anatomy is that there are few visible landmarks on the surface, but even more challenging is that two-dimensional cross-sectional images must be translated into the three-dimensional human liver.

The French surgeon and anatomist Claude Couinaud, and he developed an anatomical model that has been considered the basis of understanding liver anatomy. The Couinaud model is based on the portal veins, which generate the right and left hemilivers. The hemilivers are divided into sectors, and the sectors are divided into segments. Thus, the liver consists of 8 segments. In the right hemiliver exist segments 5 to 8 , and in the left hemiliver exist segments 2 to 4 . Segment 1 is located between the inferior vena cava and the portal vein bifurcation [72].

The Couinaud model was further developed to the now widely accepted Brisbane classification. For the second-order division, the term section was adopted, while the term segments remained for the third-order divisions. According to the Brisbane classification, the left lateral segment constitutes segments 2 and 3 , the left medial section constitutes segment 4 , the right posterior section constitutes segments 6 and 7 , and the right anterior section constitutes segments 5 and 8 [81]. 


\section{Liver imaging}

In liver surgery, preoperative imaging is of paramount importance to detect all lesions and to plan the resection. The development of different imaging modalities has rapidly increased. The importance of follow-up and early detection of eventual liver metastases was acknowledged in parallel with the advances in liver surgery $[82,83]$.

There have been several meta-analyses comparing the different imaging modalities. It has become recognized that magnetic resonance imaging (MRI), with liver-specific contrast, and especially combined with diffusion-weighted imaging, is the preferred modality due to its high sensitivity in detecting even small CRLMs [84-90].

Despite the high sensitivity of MRI with liver-specific contrast, intraoperative ultra sound (US) is still important since it may detect CRLMs not detected on MRI and hence change the planned treatment strategy [91].

It is well recognized that preoperative chemotherapy may be hepatotoxic and induce changes to the liver parenchyma, making it more difficult to accurately diagnosis the disease and hence to plan the surgical strategy [92]. A meta-analysis comparing computed tomography (CT), positron emission tomography (PET) and MRI revealed that MRI had the highest sensitivity [93].

\section{Resectability}

Approximately $70 \%$ of patients are at the time of diagnosis of CRLM not assessed as eligible for resection. The reason may be an extensive tumor burden in the liver or unresectable extrahepatic disease or that the patient is not suitable for liver resection, due to either a low performance status or comorbidities [39].

The general consensus today is that patients with CRLM should be considered for liver resection, provided that radical resection is possible, that at least two adjacent liver segments remain and that 
the patient status allows for liver surgery [94]. It is also generally acknowledged that patients with CRLM should be managed by a multidisciplinary team consisting of liver surgeons, medical oncologists and radiologists [45].

\section{Liver regeneration}

The liver is the only solid organ in the human body with the ability to regenerate and regain function after a substantial part has been resected. The ability to regain volume and function after major liver resection is crucial for the patient to recuperate. The regeneration process is complex and involves a broad range of growth factors. The majority of models studying liver regeneration after liver resection are based on animal models and may therefore not completely translate to human liver regeneration and may not fully explain the regeneration process after associating liver partition and portal vein ligation for staged hepatectomy (ALPPS) or PVO.

Liver regeneration can broadly be divided into the following phases: initiation stage, proliferation stage and termination stage. During these phases, the regeneration process aims to restore the liver volume, structure and function [95].

After major liver resection, the regeneration process is believed to be initiated with the increased flow of portal blood to the remaining part of the liver, which results in a migration of factors associated with gene transcription, such as $\beta$-catenin, to the hepatocyte nuclei. The exact mechanism by which the increase in portal blood flow starts the process is not fully understood [96].

At the initiation stage, there is also an increase in proinflammatory cytokines, of which tumor necrosis factor (TNF) and interleukin 6 (IL-6) have been found to be important in the regulation of transcription factors $[95,96]$.

During the proliferation phase, hepatocyte proliferation is stimulated by growth factors, of which hepatocyte growth factor (HGF) and epidermal growth factor (EGF) are important. Activation of HGF 
follows remodeling of the extracellular matrix, which results in the activation of HGF. HGF is a transcription factor for hepatocytes [96]. EGF, which is a growth factor found in many tissues, including the liver, is synthesized in the duodenum and moves to the liver via the portal vein. An increase in norepinephrine in plasma follows hepatectomy. Norepinephrine results in increased synthesis of EGF and contributes to activation of the HGF receptor [96].

Equally important as the proliferation phase is the termination phase. Transforming growth factor beta (TGF- $\beta$ ) is believed to terminate mitosis and the return of hepatocytes to the quiescent $G$ phase via interaction with its receptor [95].

The regeneration process starts with hepatocytes and in the periportal location and proceeds to the pericentral location in the hepatic lobule [96]. The different cell types reciprocally stimulate regeneration $[95,96]$. Kupfer cells, which are hepatic macrophages and hence inflammatory cells, promote the proliferation of hepatocytes via the synthesis of TNF and IL-6. Hepatic stellate cells and sinusoidal endothelial cells are both stimulated by the inflammatory response. Both cell types synthesize HGF and participate in the remodeling and stabilization of regenerating hepatic tissue [95].

Replication starts within 24 hours after liver resection but is not completed for another 3 to 6 months in humans. The regeneration process may be impaired in a damaged liver, for example, in steatohepatitis [95] or cirrhosis. In the complex process of liver regeneration, no single factor has been found to be necessary on its own, and all are important and cooperate through complex mechanisms [96].

The regeneration process appears to differ after ALPPS and two-stage hepatectomy (TSH), which was recognized from a clinical perspective given the faster and higher degree of volume increase after ALPPS compared to after TSH. It has been shown in animal studies that growth factors, including HGF, are higher in ALPPS groups than in PVL groups $[97,98]$. Furthermore, hepatocytes enter the cell 
cycle earlier after ALPPS than after PVL and hepatectomy, and the signaling pathways that regulate transcription may differ in ALPPS [99].

Some clinical factors may potentially decrease the regeneration capacity after major liver resection. Higher age (defined as above 65 years) has in some studies been shown to be associated with a decreased regeneration capacity [100], whereas others could not demonstrate such a correlation [101]. Most likely, age alone is not the only deciding factor, and comorbidities and liver disease could also influence the regeneration capacity.

Data regarding the impact of obesity on the regeneration capacity of the liver are conflicting. In some studies, no connection between obesity and impaired regeneration, expressed as the KGR, was found [102], whereas others found an increased risk for lower postoperative volume, expressed as the RLV/TLV for obese patients [103]. However, volume was used as a surrogate of regeneration capacity and may therefore not fully reflect the ability. Furthermore, the regeneration capacity is also most likely associated with the eventual occurrence of liver steatosis and steatohepatitis, as well as the size of the resection.

Sarcopenia, which is a depletion of skeletal muscle mass, is more commonly associated with advanced age and/or chronic disease. Sarcopenia has gained interest in recent years as a factor associated with worse outcome after liver resection. Sarcopenia is also associated with decreased volume increase after PVE $[104,105]$.

The process that regulates liver regeneration after liver surgery is complex, not fully understood and affected by a variety of factors, indicating that patient selection and preoperative planning are crucial in the attempt to decrease the risk of insufficient regeneration and thereby PHLF. 


\section{Volume of the future liver remnant}

Some patients are not assessed as eligible for resection due to the low volume of the FLR. Major hepatectomy in these cases would result in a too large of a risk of postoperative morbidity, including PHLF, and mortality.

There are different definitions of PHLF. One broadly accepted definition is the one established by the International Study Group of Liver Surgery (ISGLS). In this definition, it is concluded that if the international normalized ratio (INR) and bilirubin values have not returned to the normal preoperative values on postoperative day five, liver failure exists [106]. Another accepted definition is called the $50-50$ criteria. In this definition, liver failure exists if bilirubin is higher than $50 \mu \mathrm{mol} / \mathrm{L}$ and the prothrombin level is below $50 \%$ of the normal value on postoperative day five. PHLF, according to the 50-50 criteria, increases the risk of postoperative mortality to $59 \%$, compared to 1 $\%$ if no PHLF exists [107].

With a low postoperative volume of the FLR, up to $23 \%$ of patients suffer a major postoperative complication, including PHLF, with a 90-day mortality of up to $6 \%$. The risk is increased for those with a postoperative volume of the FLR below $20 \%$. In patients with normal livers, no increased risk was observed between those with a postoperative volume between 20 and $30 \%$, compared to those with a volume over $30 \%[108,109]$

For patients with CRLM who are treated with at least 6 cycles of preoperative chemotherapy, the risk of PHLF is increased for those with a volume below $40 \%$ [110]. For patients treated with over 12 weeks of chemotherapy, the risk of PHLF increased if the volume of the FLR was below $30 \%$ [60].

The volume limit has varied over time and varies with the eventual occurrence of compromised liver function and, to a certain extent, also between different hepatobiliary centers. In some early reports, the volume of the FLR was assessed as too low for resection upfront if it was lower than 45 $\%$ of the total liver volume, and PVE was performed before resection. The volume was calculated 
from cross-sectional imaging [111]. During the same time period, a different center estimated the volume based on body surface area (BSA) and the volume of the FLR was assessed as too low for resection up front if it was below $25 \%$ [112].

The estimation of liver volume and the volume of the FLR are not equivalent. The liver volume can be measured directly from cross-sectional imaging. The volume of the FLR is calculated from the nontumoral total liver volume [113]. Another commonly used technique to estimate TLV (total liver volume) is based on the body surface area [114]. The volume of the FLR is calculated from crosssectional imaging and yields the standardized future liver remnant (sFLR). These two estimates can differ by over $5 \%$ in approximately one-third of patients [115].

It is now a general consensus that the volume of the FLR is too small to be resected up front if it is below $20 \%$ in a normal liver, below $30 \%$ in steatosis and steatohepatitis and after chemotherapy, and below $40 \%$ in cirrhosis [116].

The relationship between volume increase and time is demonstrated by the concept of the kinetic growth rate (KGR). The KGR is defined as the increase in sFLR divided by the number of weeks between PVE and volume evaluation [117]. The volume increase in the FLR the first weeks after PVO has not been previously studied. 


\section{Techniques to increase the volume of the future liver remnant}

\section{Two-stage hepatectomy}

The term TSH was initially used to describe a strategy for patients with advanced, bilobar CRLM and for whom resection at one stage would result in a too low volume of the FLR and hence liver failure. During the first procedure, all tumors in the FLR are resected. After an interval during which the FLR regains sufficient volume, the second resection is performed, which generally constitutes a major hepatectomy [118], and for some patients, PVO was added [118, 119].

For TSH, including PVO if indicated, over $70 \%$ of patients complete both stages of TSH $[120,121]$. The majority of patients that do not proceed to the second intervention cannot do so because of tumor progression $[120,121]$. The interval between intervention 1 and intervention 2 was up to 4.5 months [120]. For those who completed both interventions, the 3-year survival was between 45 and $58 \%[120,122]$.

$\mathrm{TSH}$, without techniques for augmentation of the volume of the FLR, is now seldom practiced. Interval chemotherapy, with chemotherapy administered in the interval between intervention 1 and intervention 2, is even rarer [123].

\section{Portal vein embolization}

PVE was first described in the 1980s and was applied in patients with Klatskin tumors (liver hilar cholangiocarcinoma). The technique was developed after the observation that patients with a low volume of the FLR were at risk of developing liver failure and that patients with tumor growth in the portal vein and the lobe that required resection had an increase in the volume of the FLR [124].

PVE can be performed with different embolic agents, and none has been clearly shown to be superior in terms of an increase in the volume of the FLR and resection rate. Furthermore, most 
studies are retrospective in nature, often comparing just two or three different agents and including patients with primary tumors as well as metastatic disease.

The embolic agent can be administered as a liquid, as in the case of $\mathrm{N}$-butyl-cyanoacrylate (NBCA) or ethylene vinyl alcohol (EVOH), or in microparticles, as in the case of polyvinyl alcohol (PVA). PVA can be combined with a liquid agent such as lipiodol. Both liquid and microparticle forms can be combined with plugs such as Amplatzer vascular plugs. PVE can also be accomplished by coils.

In one study, lipiodol combined with PVA and EVOH resulted in a higher KGR than PVA combined with an Amplatzer plug, although the difference in the increase in volume was not significant [125]. Another study found that NBCA combined with lipiodol was more effective than PVA combined with coils [126]. PVA combined with coils was also inferior to NBCA combined with an Amplatzer plug [127]. However, another study found that NBCA resulted in a higher volume increase than microparticles combined with coils [128]. No study found any difference in the complication rate after PVE or the resection rate. It is therefore difficult to draw a firm conclusion regarding which agent or combination of agents is superior.

The use of PVE has also been adopted for patients with CRLM, either in the setting of two-stage resection or prior major hepatectomy. In the latter case, PVE is initially performed for patients with an estimated volume of the FLR below $35 \%$ [129]. The volume of the FLR in which PVE was applied decreases, thereby resulting in an increase in the number of patients potentially eligible for resection.

The indication for PVE is a low volume of the FLR, and resection without augmentation of the volume of the FLR would thereby bring a substantial risk of PHLF. A general consensus is that if the volume of the FLR is below $30 \%$ and the patient has been treated with preoperative chemotherapy, PVE is indicated [130]. 
PVE is generally performed percutaneously, ipsilaterally or contralaterally to the portal vein that is to be embolized. The technical success rate is reported to be between 98 and $100 \%$ [131-133]. The complication rate after PVE is generally low, and complications are not severe for the majority of patients [133]. However, up to $3 \%$ of patients suffer a complication precluding the planned hepatectomy $[131,132]$.

Evaluation of the volume increase after PVE is performed after 4 to 8 weeks $[132,134]$, and the interval between PVE and resection is from approximately 7 to 9 weeks $[131,135]$. The volume of the FLR, expressed as the standardized FLR (sFLR), increases from approximately 25 to $40 \%$ [131, 135]. SFLR is the percentage volume of the total liver volume, which is calculated from a formula based on body surface area (BSA) [114].

The resection rate after PVE is approximately 63 - $76 \%$ [131-133]. For patients undergoing PVE before radical hepatectomy as the only surgical procedure, the resection rate is higher than that for those undergoing TSH [134]. Up to $63 \%$ of the patients who do not proceed to radical hepatectomy are unable to do so due to tumor progression [132]. If chemotherapy is stopped some weeks before PVE, up to $68 \%$ of patients have tumor progression, and over half of those patients are unable to proceed to radical hepatectomy [136]. However, if chemotherapy is administered in the interval between PVE and resection, the outcome is improved without preventing a sufficient volume increase [137]. Approximately 6 to $9 \%$ of patients have an insufficient volume increase to proceed to radical hepatectomy $[132,136]$.

The long interval between PVE and radical hepatectomy is to ensure a sufficient volume increase in the FLR. However, data from studies of liver function indicate that function precedes the volume increase [138]. It would therefore be possible to shorten the interval and enable more patients to proceed to radical hepatectomy. 
The three-year survival for patients with CRLM resected after PVE was 54 to $67 \%[131,132]$, and the three-year DFS was $14 \%$ [132]. For patients who experience progression after PVE, but can still proceed to intervention 2, they had a similar long-term outcome as those with stable disease [136].

The need for PVE due to a low volume of the FLR does not seem to result in worse long-term survival. In addition to a low volume of the FLR, undergoing major hepatectomy, including PVE for those with a low volume of the FLR, can result in a 5-year survival of $43 \%$ for those treated with PVE compared to $42 \%$ for those with sufficient volume of the FLR treated with upfront hepatectomy [135]. There is no difference in survival if the analysis is performed considering an intention to treat principle or considering bilobar disease in patients. The resection rate in the PVE group was $80 \%$, and the median survival in the PVE group was 45 months, compared to 49 months in the group resected up front [139]. This result was confirmed by a matched comparison of patients resected after PVE with patients who resected upfront, as a 5-year survival of $14 \%$ was seen in both groups [140].

\section{Portal vein ligation}

Data showing that ligation of a branch of the portal vein subsequently results in increased volume of the nonligated lobe were published nearly 100 years ago [141]. It then took almost 80 years before PVL became a recognized technique to increase the volume of the FLR before major liver resection [142]. One of the first studies of PVL and TSH in patients with advanced liver metastases was published in 2003 [143].

There have been some questions regarding whether PVL is as effective as PVE for inducing a volume increase in the FLR $[144,145]$, but studies have concluded that PVL results in a similar increase in the volume of the FLR as PVE [146]. 


\section{Concerns regarding portal vein occlusion}

One of the main concerns regarding PVO is the relatively long interval between PVO and radical hepatectomy. Consequently, a rather high proportion of patients do not reach the second stage, mainly due to progression or an insufficient volume increase in the FLR $[17,132,147]$.

\section{Associated liver partition and portal vein ligation for staged hepatectomy}

The first article describing ALPPS was published in 2012 [148], and the first ALPPS procedure was performed in 2007. In this first published article, the procedure was performed on patients with primary hepatobiliary tumors as well as on patients with metastatic disease, including CRLM. All patients had tumors requiring extended right hepatectomy to achieve radical resection. On the preoperative volumetric evaluation, the FLR/body weight ratio was below 0.5 . During the first intervention, the right portal vein was divided, and the parenchyma was completely or nearly completely transected. After a median of 9 days, the volume of the FLR had increased $74 \%$. During the second intervention, the artery and bile duct leading to the right lobe were transected, and the hepatectomy was completed. For some patients (40\%), the biliary system was reconstructed with hepaticojejunostomy. All patients had sufficient volume increase, and all patients proceeded to the second intervention. During the postoperative course, no patient experienced PHLF. The total frequency of complications was $64 \%, 44 \%$ of the complications were grade III or IV according to the Clavien-Dindo classification, and the mortality rate was $12 \%$. The median follow-up was rather short, 180 days, and the estimated median survival at 6 months was $86 \%$ [148].

The ALPPS procedure rapidly gained interest, and in 2012, the ALPPS registry was initiated (www.alpps.net). The purpose of the registry was to centralize data for all patients undergoing ALPPS. The registry serves to centralize collected data for all patients undergoing ALPPS worldwide. 
After the first description of ALPPS, several centers adopted the technique, and the number of publications rapidly increased. Most were retrospective studies; the indication for ALPPS was a low volume of the FLR, and ALPPS was conducted for patients with primary hepatobiliary tumors as well as metastatic disease. The preoperative volume of the FLR ranged from 23 to $28 \%$ [149-152], and the volume of the FLR increased between 40 and $87 \%$ from days $6-8$ [149-153]. The interval between intervention 1 and intervention 2 ranged between 7 and 13 days [149, 150, 152, 153]. In the initial reports, all patients completed both interventions. The overall complication rate was between 33 and $67 \%$, including a mortality rate between 0 and $29 \%$ [149-153].

The technique evolved, and less invasive methods were proposed to decrease postoperative morbidity and mortality while still aiming to achieve the same volume increase and resection rate.

Compared with traditional ALPPS, partial ALPPS, in which the parenchyma transection during intervention 1 is not completed, resulted in an equal volume increase and resection rate but with no postoperative mortality and a decreased complication rate [154].

Associating liver tourniquet and portal ligation for staged hepatectomy (ALTPS) was suggested to be a less invasive method. In ALTPS, a tourniquet is placed in the planned transection line during intervention 1 instead of transection of the parenchyma. ALTPS seemed to result in a similar volume increase as ALPPS, but in the initial reports, the complication rate was similar, including the mortality rate [155]. Other minimally invasive versions of ALPPS have been described. Some are completely laparoscopic, including transection of the parenchyma [156]. Other techniques consist of either microwave or radiofrequency ablation along the planned transection line $[157,158]$. There has also been a description of patients undergoing robotic ALPPS [159].

The indications for ALPPS expanded to include patients with an insufficient volume increase after PVO (rescue ALPPS). The studies consisted of rather small series of patients with only CRLM [160, $161]$ or, in one study, or with other diagnoses than as well as CRLM $[162,163]$. 
Patients with potential eligibility for resection were further included with the description of monosegment ALPPS, in which the FLR consisted of a single liver segment [164].

The results regarding oncological results for some of the first patients undergoing ALPPS showed that the median OS was 26 months in a series of patients with primary hepatobiliary tumors as well as metastatic disease including CRLM. The survival for patients with CRLM was higher than that for patients with other diagnoses, although the median survival was not stated for the subgroups [165].

The first large study regarding perioperative morbidity and mortality, risk factors for complications and oncological outcome was based on data from the ALPPS registry [166]. In this study, it was shown that there was a difference in outcome depending on diagnosis and age; patients younger than 60 years and patients with CRLM had better long-term outcomes than older patients and patients with other diagnoses. It was also found that risk factors for severe complications were diagnoses other than CRLM, age over 60 years, blood transfusion and long operating time during intervention 1 . Furthermore, the overall postoperative mortality was shown to be $9 \%$, indicating an improvement in patient selection in the participating centers [166].

The focus on and interest in expanding indications, improving patient selection and reducing perioperative morbidity and mortality have increased. Another study based on data from the ALPPS registry revealed that the indication had shifted increasingly toward CRLM, and the patient population shifted towards younger patients. [167]. Furthermore, the interval between intervention 1 and intervention 2 increased, and complications in the interval between stage 1 and stage 2 became recognized as a factor determining the risk of mortality after ALPPS $[167,168]$.

There were concerns that a rapid increase in volume may not be correlated to an equal increase in function, which could, at least partly, explain the higher morbidity for ALPPS than for TSH. This possibility was highlighted in that the increase in the volume of the FLR exceeded the increase in function [169-171]. Furthermore, data indicated that the total liver function was lower between intervention 1 and intervention 2 due to reduced function in the deportalized lobe [172]. 
The long-term oncological result related to ALPPS remained less certain than those related to TSH, and there have been several retrospective analyses of ALPPS and TSH. Some included only patients with CRLM [173-175], whereas some included patients with primary hepatobiliary tumors as well as metastatic disease [176]. The resection rate was higher for patients treated with ALPPS than for those treated with TSH [174-176]. Regarding complications, some studies found a higher complication rate after ALPPS than after TSH $[173,176]$, whereas in other studies, no difference could be found [174, 175]. In the survival analysis, some studies demonstrated improved survival for patients treated with TSH, but in the analysis, only patients who completed both interventions were included [174]. Other studies could not find a difference in OS but did find a shorter DFS for patients treated with ALPPS than for those treated with TSH $[173,175]$.

There was also a comparison between ALPPS and palliative chemotherapy for patients with advanced CRLM. The survival for patients undergoing ALPPS was longer, but the difference did not reach statistical significance [177].

Although there is still debate as to whether ALPPS has a role in the treatment of patients with advanced liver tumors, the general consensus has increasingly recognized that ALPPS is most suited for patients with CRLM and that the resection rate of ALPPS is higher than that of TSH. There is still some uncertainty regarding the effect of preoperative chemotherapy on the volume increase and the postoperative outcome. Whether ALPPS has superior in oncological outcomes remains unanswered. The data thus far are based on retrospective analyses and single center studies. In the first RCT randomizing patients with advanced CRLM to ALPPS or TSH, it was shown that the resection rate for patients randomized to ALPPS was significantly higher than that for patients randomized to TSH and that there was no significant difference in the complication rate, as well as no difference in mortality [178]. 
After the introduction of ALPPS as a technique for patients with a low volume of the FLR and given the initially high complication rate for patients treated with ALPPS, there was a retrospective analysis of PVE compared to ALPPS in which the authors argued that PVE was superior to ALPPS in terms of a lower complication rate and a relatively favorable long-term outcome [147]. However, it was a retrospective study comparing data from one center with previously published data. Furthermore, diagnoses other than CRLM were included in both groups. Finally, the authors did not consider that ALPPS was at the time a relatively new method, whereas PVE was a well-developed technique.

\section{Other techniques to achieve radical resection for patients with advanced colorectal liver}

\section{metastases}

The resection rate is high for patients treated with ALPPS, but due to the still rather high postoperative morbidity, ALPPS has been criticized, and there have been efforts to develop other less invasive methods.

One drawback with PVE is that a relatively large proportion of patients will not proceed to radical hepatectomy, either due to tumor progression during the interval between PVE and resection or due to an insufficient volume increase in the FLR.

One technique that has been proposed to be associated with a lower frequency of postoperative complications is enhanced one-stage ultrasound-guided hepatectomy (e-OSH). e-OSH consists of the following principles: the tumors are located with ultrasound guidance; R1 resection is performed for those tumors that are located close to large blood vessels; hepatic vein resection and reconstruction are performed when a tumor marginally infiltrates the vein; and finally, the outflow is preserved. In one retrospective study comparing the outcomes of patients treated with ALPPS and e-OSH, the OS did not differ between patients treated with ALPPS and patients treated with e-OSH. The number of 
patients who received R1 resection was higher in the e-OHS group, and the DFS was shorter, but the differences did not reach statistical significance [179].

Other techniques, which are similar to and sometimes considered variations of ALPPS, consist of either microwave or radiofrequency ablation performed along the planned transection line (RALPPS) instead of transection of the parenchyma $[157,158]$. Some studies have indicated that RALPPS results in a significantly higher resection rate than PVE without an increased complication rate [180]. 


\section{Health economics}

Health economics is a subdiscipline of economics and started to emerge as a separate discipline during the 1960s. One of the first articles on the subject was written by Kenneth J Arrow, a professor of economics at Stanford University, USA. In one article, he discussed how the economics of the medical care industry are unique compared with other types of economics [181]. It was, however, recognized approximately a century earlier by Karl Marx that a relationship existed between the economic status of an individual and their health [182].

Health economics analyzes the economic aspects of health care and can also influence decisions on health politics. Health economics also addresses the issue of how much societal members are prepared to pay to achieve a certain level of health. Furthermore, health economics considers how the limited resources of health care systems can be allocated and used in health care [183].

Since the 1960s, health economics has been applied to study the effect of interventions, for example, pharmacological or surgical interventions, on health as well as on the cost of health care. In health economics, two alternative treatments for the same condition are compared in terms of outcome and cost. The analyses are performed systematically, and a new treatment is compared to an old treatment. The costs as well as the effects can be combined in the analyses and are often considered as an incremental cost effectiveness ratio (ICER). The difference in costs for two alternative treatments is divided by the difference in effect by the same treatments [184].

$$
\text { ICER }=\text { Cost treatment } A^{-} \text {Cost treatment } B / \text { Effect treatment } A^{-} \text {Effect treatment } B
$$

The effect of the treatment is often expressed as either the quality of life years (QALYs) or life years (LYs). In both estimates, survival is included from a predefined starting point until the end of followup [185]. To estimate quality of life, a questionnaire is often used, which can be disease-specific or generic [186]. 
One commonly used questionnaire is the EQ-5D, which was developed during the 1980s [186]. The intention was to develop a tool that was not disease-specific that could be used internationally, and the EQ-5D has been validated and translated into Scandinavian languages. The questionnaire includes 5 different dimensions. These are mobility, self-care, ability to perform usual activities, pain/discomfort and anxiety/depression. For each dimension, there are 3 levels, ranging from no symptoms/problems to severe symptoms/problems. Hence, there are 243 possible health states [187].

When QALYs are used, the analysis is described as a cost utility analysis (CUA), and when LYs are used, the analysis is described as a cost effectiveness analysis (CEA) [188].

\section{Health economic evaluation of patients with colorectal liver metastases}

There are relatively few previous health economy analyses of patients with CRLM undergoing resection. The number is even more scarce regarding patients with advanced disease undergoing ALPPS, and there is no comparison of the health economic outcome for patients randomized to ALPPS or TSH.

For patients with CRLM and different tumor burdens in the liver undergoing resection, with methods ranging from wedge resection to hemihepatectomy, it was found that the HRQoL was reduced in the early postoperative period but improved, and after 6 months, it was almost restored to the preoperative level [189]. However, there was no subgroup analysis during the follow-up, and it is therefore not possible to conclude whether there was any difference in HRQoL for patients depending on the extent of the resection. Neither was it possible to conclude whether the HRQoL differed between patients with recurrent disease and those who remained tumor free.

For patients with CRLM undergoing ALPPS, one previous study evaluated HRQoL. The evaluation was performed approximately 2 years after intervention 2, and the HRQoL at that time was comparable with the QoL for the general population. No difference in QoL could be found for those with 
recurrent disease [190]. The number of patients alive at the time of the evaluation was, however, relatively small, and the recurrence rate was rather high, with some $79 \%$ of the patients experiencing recurrence during the follow-up, which was a median of 35 months. This could indicate that the majority of the patients had recurrent disease at the time of the evaluation. Furthermore, the questionnaire was completed via telephone, and a possible bias toward a higher rating cannot be excluded.

There are some data indicating that resection of CRLM is more cost effective than palliative treatment, including palliative chemotherapy $[191,192]$. For patients treated with resection, adjuvant chemotherapy was however not included in the analysis In another model, resection was also shown to be more cost effective if postoperative follow-up and resection of eventual recurrent disease were included in the analysis [193].

Accordingly, available data indicate that resection is more cost effective than palliative treatment and that QoL after liver resection may return to the preoperative level. Given the limited resources in health care systems, the evaluation of costs and outcomes is of paramount importance. 


\section{Aims of the thesis}

The overall aim of the thesis was to study different aspects of and outcomes after surgical treatment for patients with advanced CRLM treated with ALPPS or TSH.

Specific aims for studies I-IV

I. To study whether preoperative chemotherapy had a negative impact on the volume increase for patients undergoing ALPPS.

II. To study the temporal volume increase of the FLR after PVO.

III. To study the oncological outcome for patients with CRLM randomized to ALPPS or TSH.

IV. To study the health economic outcome for patients with CRLM randomized to ALPPS or TSH. 


\section{Material and methods}

\section{Ethical permissions}

Study I was approved by the Cantonal Ethics Committee, Zurich, Switzerland, (KEK 2013-0326).

Study II-IV was approved by the Regional Ethics Committee, Gothenburg, Sweden, (ref 098-14).

\section{Study I}

Study I was based on data from the ALPPS registry (www.alpps.net). The registry started in 2012, with the intent to collect data in a standardized dataset for patients undergoing ALPPS. In study 1 , 442 patients from 91 centers from 30 countries participated. All patients with CRLM who were registered from the start of the registry until May 13, 2016 were included. The inclusion criteria were CRLM, and the exclusion criteria were diagnoses other than CRLM.

In the registry, the data were organized into the following main categories: demographics, current disease, comorbidity, intervention 1, intervention 2, liver function and postoperative complications during the hospital stay. From the registry, it was possible to obtain data on which liver segments were affected by the tumor and which segments were part of the FLR. It was also possible to obtain data regarding the surgical procedures and whether wedge resections were performed due to metastases in the FLR.

To calculate the time for the volume increase in the FLR, the number of days between intervention 1 and the last radiological examination before intervention 2 was used. The volume increase was calculated in three ways: as the absolute increase, expressed in milliliters; as the percentage increase; and as the KGR. For all methods, the clean volume of the FLR was used, which is the tumorfree volume of the FLR. The formula for calculating the KGR was from a previously published method 
[117]: $K G R=D H /$ number of weeks. $D H$ was calculated by subtracting $s F L R_{\text {pre }}$ from $s F L R_{\text {post }}$. The number of weeks was the duration from PVO to the volume evaluation.

The study population was divided into four groups based on preoperative chemotherapy: no preoperative chemotherapy; one chemotherapy regimen; more than one chemotherapy regimen; and chemotherapy and monoclonal antibodies.

\section{Statistical method study I}

To compare the volume increases for patients in the four different groups, analysis of variance was used. To evaluate the eventual effect of covariates, analysis of covariance was used. The following factors were covariates: age, body mass index, duration of preoperative chemotherapy, Charlson comorbidity index, sex and complications after intervention 1.

\section{Study population study II-IV}

The study population in studies II - IV consisted of patients included in the Scandinavian randomized controlled trial LIGRO (ClinicalTrials.gov, NCT02215577). Six hepatobiliary centers from Sweden, Norway and Denmark participated. In the LIGRO trial, patients with CRLM and low volume of the FLR were randomized to ALPPS or TSH. The inclusion criteria were $\mathrm{CRLM}, \mathrm{SFLR}<30 \%$, at least stable disease on preoperative chemotherapy and resectability with a two-stage approach. The exclusion criteria were liver cirrhosis, age below 18 years and severe comorbidity. Extrahepatic disease was not an exclusion criterion if assessed as resectable. Patients with progressive disease on preoperative chemotherapy at evaluation were excluded. One hundred patients were included, and 50 patients each were randomized to ALPPS and TSH. Two patients randomized to ALPPS were excluded due to severe comorbidity and sFRL $>30 \%$. One patient randomized to TSH was excluded due to progressive disease. 
For all patients randomized to ALPPS, classic ALPPS was performed, which indicates that complete transection of the parenchyma was performed at intervention 1.

There was no formal criteria in the study protocol for patients randomized to TSH and for whether they were treated with PVE or PVL. For patients treated with PVE, the procedure was performed according to the practice at each center and was consistent during the study period. Thirty-five patients underwent PVE, and fourteen patients underwent PVL. Rescue ALPPS was accepted within the protocol for patients randomized to TSH and with insufficient volume increase at radiological evaluation.

Volume evaluation was performed with either CT or MRI according to the discretion and clinical routine of each participating center.

Patients with metastases in the FLR were treated with either ablation or wedge resection, in conjunction with either intervention 1 or intervention 2, at the discretion of the participating center. The date of diagnosis of liver metastasis was determined as the date of the first regional tumor board before the start of neoadjuvant chemotherapy.

\section{Follow-up study III and IV}

The follow-up time in the protocol was established as 2 years after the last intervention. Follow-up after this time period occurred according to clinical practice at each participating center and was therefore not included in the analysis in study IV. The first follow-up was approximately 4 weeks after the last intervention and then after approximately 4, 8, 12, 18 and 24 months. At the first follow-up, adjuvant/palliative chemotherapy was considered. For those patients with the primary tumor not resected prior to liver surgery or with EHD, resection was planned, provided that the patient was suitable for an additional surgical procedure. 
At each time point, either CT or MRI was performed, and the tumor status was noted. A patient was assessed as tumor free (if no tumor was evident on the radiological examination), as having recurrence or as having residual tumor. For patients with recurrent disease, resection, ablation or palliative chemotherapy was considered according to tumor burden and the general condition of the patient.

\section{Statistical methods study I-IV}

The results are expressed as the mean \pm standard deviation or as the median (range), as appropriate. Continuous data were compared with a $t$-test or analysis of variance, and categorical data were compared with the chi-square test.

\section{Study II}

The study population in study II consisted of patients randomized to TSH. The first radiological evaluation of the volume increase was performed within the first week after PVO. For those with insufficient volume increase, additional evaluation was performed within 2 to 4 weeks, depending on the volume of the FLR and the KGR at the first evaluation. The radiological evaluation was performed with either CT or MRI. The volume of the FLR was expressed as the SFLR. To estimate the volume increase, the KGR was used according to a previously published formula [117]: KGR = $\mathrm{DH} /$ number of weeks. DH was calculated by subtracting the $s F L R_{\text {pre }}$ from the $s F L R_{\text {post. }}$. The KGR was calculated over the duration from PVO to last evaluation before radical hepatectomy but also over the intervals between PVO and radiological evaluation and between radiological evaluations. 


\section{Statistical method study II}

The KGR was expressed as \%/week. A $t$-test or ANOVA was used to compare continuous data, and post hoc analyses were performed with Bonferroni correction. For categorical data, the chi-square test was used. A p-value $<0.05$ was considered statistically significant.

\section{Study III}

The first patient was included in August 2014, and the last patient was included in June 2016. All patients had an at least two-year follow-up. The date for censoring survival was between September and December 2018 because the LIGRO trial was a multicenter study.

\section{Statistical method study III}

To estimate the length of follow-up, a reversed Kaplan-Meier method was used, and the starting point was the date of ALPPS intervention 1 or PVO. To estimate survival, the Kaplan-Meier and logrank tests were applied. Survival analysis was performed both according to the intention-to-treat (ITT) principle and per protocol (PP). In the ITT analysis, all randomized patients were included, and in the PP analysis, the patients who underwent liver resection were included. As the starting point in the survival analysis, the date of diagnosis of CRLM and the date of the first intervention were used. Disease-free survival was assessed from the date of the surgical procedure by which the patient was rendered tumor free until the date of recurrent disease.

Subgroup survival analysis was performed for patients randomized to TSH but treated with rescue ALPPS and for patients in both groups that did not undergo radical hepatectomy.

Cox regression analysis was performed in the survival analysis. In the multivariable Cox regression analysis, factors with $p$-values $<0.1$ in the univariable analysis were included. 


\section{Study IV}

\section{Economic evaluation}

Resource use, costs and health outcomes were compared for all patients randomized to ALPPS or TSH. The starting time for analysis was the date of the first intervention (ALPPS intervention 1, PVE or PVL) and continued for two years or until death, whichever came first.

Cost-effectiveness analysis was performed. In this analysis, the incremental health care costs were related to the incremental health outcome for patients randomized to ALPPS compared to patients randomized to TSH. The reported incremental cost-effectiveness ratio (ICER) should be considered the additional cost of achieving additional health outcomes if a patient is treated with ALPPS rather than TSH [194].

The costs were reported for the entire study period but also in three intervals. Period 1 was the perioperative period, started with intervention 1 and ended 30 days after the last intervention. Period 2 continued after period 1 until 12 months, and period 3 started at 13 months and continued until 24 months.

\section{Resource use}

All surgical interventions for ALPPS and TSH were included, and the length of stay after the interventions was noted. During follow-up, resection of the primary tumor and EHD were included. During the follow-up, radiological examinations and chemotherapy were also included.

In the concept of resource use, the following interventions and events were included: surgical interventions, hospitalization, diagnostic procedures and chemotherapy. The included surgical procedures were ALPPS intervention 1 and ALPPS intervention 2 for patients randomized to ALPPS. For patients randomized to TSH, PVE, PVL and rescue ALPPS were included procedures. For both groups, ablation of metastases in the FLR and radical hepatectomy were included as well as 
postoperative complications $\geq 3 a$ according to the Clavien-Dindo classification. The microscopic examination results after ALPPS and TSH were included. Surgical procedures included during followup were resection of the primary tumor, resection of EHD or recurrent liver metastasis and ablation of recurrent liver metastasis. Regarding hospitalization, the length of stay after ALPPS/TSH, readmissions within 30 days and the length of stay after resection of the primary tumor, recurrent disease and EHD were included. Included diagnostic procedures during follow-up were CT and MRI. The type and number of cycles of adjuvant and palliative chemotherapy were included.

\section{Unit costs}

To obtain a health care cost for each included patient, the resource use was multiplied by the unit cost. Calculated and estimated costs were based on costs at Linköping University Hospital, Sweden, in 2018. The conversion to euros was based on the exchange rate set by the Swedish National Bank in October 2018 ( 1 euro = 10,71 SEK). It was assumed that the costs did not differ for the other participating countries.

The unit cost of surgical interventions was based on the mean operating time for the respective procedure. Included in the cost of each surgical procedure were the preoperative preparation, operating time in minutes, the surgical equipment required for the respective procedure, recovery at the postoperative ward and the attendance of anesthesiologist, nurses and surgeons.

The cost of the microscopic histopathological examination was based on the number and types of resections.

The unit costs of the hospitalizations were based on a fixed cost for each care event and a cost per day for care at the intensive care unit's respective surgical ward.

The unit cost of radiological examinations was based on the number and type of examinations. 
The unit cost of chemotherapy was estimated from the cost of the respective agent and the number of cycles.

\section{Health outcomes}

To assess quality of life, the EQ-5D was used. The EQ-5D was administered prior to intervention 1 , approximately 1 month after intervention 2 and then after approximately 6, 12 and 24 months. Missing data were imputed with the median value from corresponding patients at the same time of measurement: patients randomized to the same treatment and with the same tumor status. Patients who did not proceed to intervention 2 were not followed up with the EQ-5D. It was assumed that they had the same QoL as patients with recurrent disease.

The UK EQ-5D value set was applied to obtain a single value of health [195]. QALYs were calculated from the area under the curve with the assumption that a linear relationship existed between two measurement points. The calculation started at the date of intervention 1, and it was assumed that the HRQoL was the same as that at inclusion. For patients dying during the study period, the last value was assumed to decline linearly to zero at the time of death.

\section{Statistical method study IV}

All included patients were included in the analyses, and the analyses were performed according to the intention-to-treat principle (ITT). The results are expressed as the mean $(95 \% \mathrm{CI})$. The $t$-test was used for continuous data, and the chi-square test was used for comparison of categorical data. Nonparametric bootstrapping was performed to display the joint distribution of the mean incremental costs, QALYs, and life years (LYs). 


\section{Results}

\section{Study I}

During the study period, 442 patients were registered, and for 404 patients, it was stated that both interventions were undertaken. For ten patients $(2 \%)$, it was stated that they did not proceed to intervention 2, and the reason was stated for 3 patients. For two patients, it was due to complications, and for one patient, it was due to tumor progression. For the remaining 28 patients, data were lacking regarding whether they did proceed to intervention 2 or not. For a detailed description regarding preoperative clinical information and preoperative volumes, see table 1.

\begin{tabular}{|c|c|c|c|c|}
\hline & $\begin{array}{l}\text { No neoadjuvant } \\
\text { therapy }(n=45)\end{array}$ & $\begin{array}{l}\text { One regimen of } \\
\text { chemotherapy } \\
(n=185)\end{array}$ & $\begin{array}{l}\text { More than on } \\
\text { regimen of } \\
\text { chemotherapy } \\
(n=16)\end{array}$ & $\begin{array}{l}\text { Monoclonal } \\
\text { antibodies } \\
(n=196)\end{array}$ \\
\hline Age (mean $\pm 1 \mathrm{SD}$ ) & $62 \pm 12(n=43)$ & $60 \pm 10(n=182)$ & $60 \pm 10(n=16)$ & $58 \pm 12(n=196)$ \\
\hline Sex: female/male & $19 / 25$ & $69 / 114$ & $4 / 12$ & $65 / 129$ \\
\hline BMI (mean \pm 1 SD) & $27 \pm 4(n=43)$ & $26 \pm 7(n=184)$ & $26 \pm 6(n=16)$ & $26 \pm 4(n=195)$ \\
\hline $\begin{array}{l}\text { Charlson score (mean } \pm 1 \\
\text { SD) }\end{array}$ & $6 \pm 1$ & $6 \pm 1$ & $7 \pm 1$ & $6 \pm 1$ \\
\hline Synchronous presentation & $51 \%(n=23)$ & $65 \%(n=120)$ & $56 \%(n=9)$ & $80 \%(n=157)$ \\
\hline Metachronous presentation & $40 \%(n=18)$ & $21 \%(n=38)$ & $38 \%(n=6)$ & $15 \%(n=31)$ \\
\hline $\begin{array}{l}\text { Number of tumors } \\
\text { preoperatively (mean } \pm 1 \\
\text { SD) }\end{array}$ & $4 \pm 3(n=31)$ & $6 \pm 5(86)$ & $4 \pm 3(n=8)$ & $5 \pm 4(n=115)$ \\
\hline \multicolumn{5}{|l|}{$\begin{array}{l}\text { Preoperative volume of the } \\
\text { FLR }\end{array}$} \\
\hline sFRL (mean \pm 1 SD) & $21 \% \pm 7(n=35)$ & $25 \% \pm 9(n=150)$ & $21 \% \pm 7(n=15)$ & $23 \% \pm 8(n=155)$ \\
\hline $\begin{array}{l}\text { clean FLR/TLV (mean } \pm 1 \\
\text { SD) }\end{array}$ & $26 \% \pm 6(n=31)$ & $26 \% \pm 8(n=113)$ & $27 \% \pm 11(n=11)$ & $27 \% \pm 9(n=111)$ \\
\hline $\begin{array}{l}\text { clean volume FLR in } \mathrm{ml} \\
\text { (mean } \pm 1 \mathrm{SD} \text { ) }\end{array}$ & $331 \pm 110(n=36)$ & $384 \pm 152(n=153)$ & $342 \mathrm{ml} \pm 141(\mathrm{n}=15)$ & $362 \pm 135(n=161)$ \\
\hline $\begin{array}{l}\text { Duration of neoadjuvant } \\
\text { therapy in months (mean, } \\
95 \% \mathrm{Cl} \text { ) }\end{array}$ & & $5(4-6)(n=113)$ & $8(6-10)(n=13)$ & $6(5-7)(n=172)$ \\
\hline
\end{tabular}

Table 1. Clinical and preoperative volumes (study 1). sFRL: standardized future liver remnant, FLR: future liver remnant, TLV: total liver volume, SD: standard deviation, $\mathrm{Cl}$ : confidence interval 
Forty-five (10\%) of the included patients were not treated with preoperative chemotherapy. Of the patients with oncological treatment, $185(42 \%)$ received 1 regimen, $16(4 \%)$ received more than 1 regimen, and 196 (44\%) received monoclonal antibodies in addition to chemotherapy. In all three groups treated with preoperative chemotherapy, oxaliplatin-based regimens were the most frequent chemotherapy. In the group treated with monoclonal antibodies, bevacizumab was the most frequent monoclonal antibody. For a detailed description of the preoperative oncological treatment, see table 2 . 


\begin{tabular}{|c|c|c|c|c|c|}
\hline $\begin{array}{l}\text { Table with } \\
\text { neoadjuvant } \\
\text { chemotherapy }\end{array}$ & $\begin{array}{l}\text { Name of the } \\
\text { regimen }\end{array}$ & First regimen & Second regimen & Third regimen & $\begin{array}{l}\text { Fourth and fifth } \\
\text { regimens }\end{array}$ \\
\hline \multicolumn{6}{|c|}{$\begin{array}{l}\text { One chemotherapy } \\
\text { regimen }(n=188)\end{array}$} \\
\hline & FOLFOX & $48 \%(n=90)$ & & & \\
\hline & FOLFIRI & $11 \%(n=21)$ & & & \\
\hline & XELOX & $(7 \% n=13)$ & & & \\
\hline & Other* & $10 \%(n=19)$ & & & \\
\hline & $\begin{array}{l}\text { Regimen not } \\
\text { specified }\end{array}$ & $24 \%(n=45)$ & & & \\
\hline \multicolumn{6}{|l|}{$\begin{array}{l}\text { More than one } \\
\text { chemotherapy } \\
\text { regimen }(n=16)\end{array}$} \\
\hline & FOLFOX & $44 \%(n=7)$ & $6 \%(n=1)$ & & \\
\hline & FOLFIRI & $13 \%(n=2)$ & $25 \%(n=4)$ & $6 \%(n=1)$ & \\
\hline & XELOX & $13 \%(n=2)$ & $6 \%(n=1)$ & & \\
\hline & Other & $31 \%(n=5)$ & $63 \%(n=10)$ & $13 \%(n=2)$ & \\
\hline \multicolumn{6}{|c|}{$\begin{array}{l}\text { Chemotherapy and } \\
\text { antibodies }(n=196)\end{array}$} \\
\hline & $\begin{array}{l}\text { FOLFOX and } \\
\text { bevacizumab }\end{array}$ & $33 \%(n=64)$ & $2 \%(n=4)$ & & \\
\hline & $\begin{array}{l}\text { FOLFOX and } \\
\text { cetuximab }\end{array}$ & $12 \%(n=23)$ & $1 \%(n=2)$ & & $\begin{array}{l}\text { 5th regimen } 0.5 \\
\% n=1\end{array}$ \\
\hline & $\begin{array}{l}\text { FOLFOX and } \\
\text { panitumumab }\end{array}$ & $7 \%(n=13)$ & & & \\
\hline & $\begin{array}{l}\text { FOLFIRI and } \\
\text { bevacizumab }\end{array}$ & $19 \%(n=38)$ & $6 \%(n=12)$ & $1.5 \%(n=3)$ & \\
\hline & $\begin{array}{l}\text { FOLFIRI and } \\
\text { cetuximab }\end{array}$ & $11 \%(n=22)$ & $2 \%(n=4)$ & & $\begin{array}{l}\text { 4th regimen } 1.5 \\
\% n=3\end{array}$ \\
\hline & $\begin{array}{l}\text { FOLFIRI and } \\
\text { panitumumab }\end{array}$ & $0.5 \%(n=1)$ & $1 \%(n=2)$ & $0.5 \%(n=1)$ & \\
\hline & $\begin{array}{l}\text { XELOX and } \\
\text { bevacizumab }\end{array}$ & $4 \%(n=8)$ & & & \\
\hline & $\begin{array}{l}\text { XELOX and } \\
\text { cetuximab }\end{array}$ & $1 \%(n=2)$ & & & \\
\hline & $\begin{array}{l}\text { Xeloda and } \\
\text { bevacizumab }\end{array}$ & $1 \%(n=2)$ & & & \\
\hline & $\begin{array}{l}\text { Xeloda and } \\
\text { cetuximab }\end{array}$ & $1 \%(n=2)$ & & & \\
\hline & Other* & $11 \%(n=21)$ & $10 \%(n=19)$ & $7 \%(n=13)$ & $1 \%(n=2)$ \\
\hline
\end{tabular}

Table 2. Description of preoperative chemotherapy and number of patients receiving the respective agent. FOLFOX: fluorouracil, oxaliplatin and folinic acid; FOLFIRI: fluorouracil, irinotecan and folinic acid; XELOX: capecitabine and oxaliplatin

For the entire cohort, the mean interval between intervention 1 and intervention 2 was 11 days, and during this interval, FLR increased $263 \pm 139 \mathrm{ml}(82 \pm 24 \%)$, and the KGR was $15 \pm 10 \% /$ week.

The volume increase, expressed as $\mathrm{ml}$, was highest for patients treated with 1 regimen of chemotherapy and was $306 \mathrm{ml}$, compared to $301 \mathrm{ml}$ for patients not treated with chemotherapy and 
$287 \mathrm{ml}$ for the group treated with monoclonal antibodies. The volume increase was lowest for the group treated with more than 1 regimen and was $241 \mathrm{ml}$ (Figure 1).

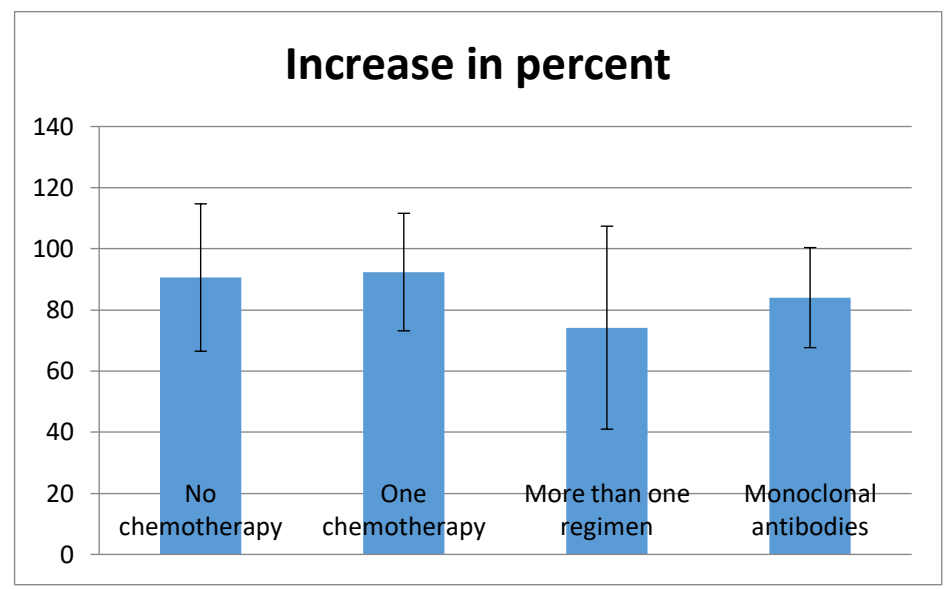

Figure 1. Volume increase of the FLR expressed as a percent. The FLR increased $91 \%$ ( $95 \mathrm{Cl} 66-115$ ) for the group not treated with chemotherapy, $92 \%$ (95 Cl 73-112) for the group treated with one regimen of chemotherapy, $74 \%$ for the group treated with more than one regimen of chemotherapy (95 Cl 41-107) and $84 \%$ (95 Cl 68-100) for the group treated with monoclonal antibodies (Study 1).

The percentage increase was highest in the group treated with 1 regimen and was $92 \%$, and the lowest increase, expressed as a percent, was seen in the group treated with more than 1 regimen and was $74 \%$. For patients treated with the addition of monoclonal antibodies, the percentage increase was $84 \%$, and in the group with no chemotherapy, it was $91 \%$ (Figure 2). 


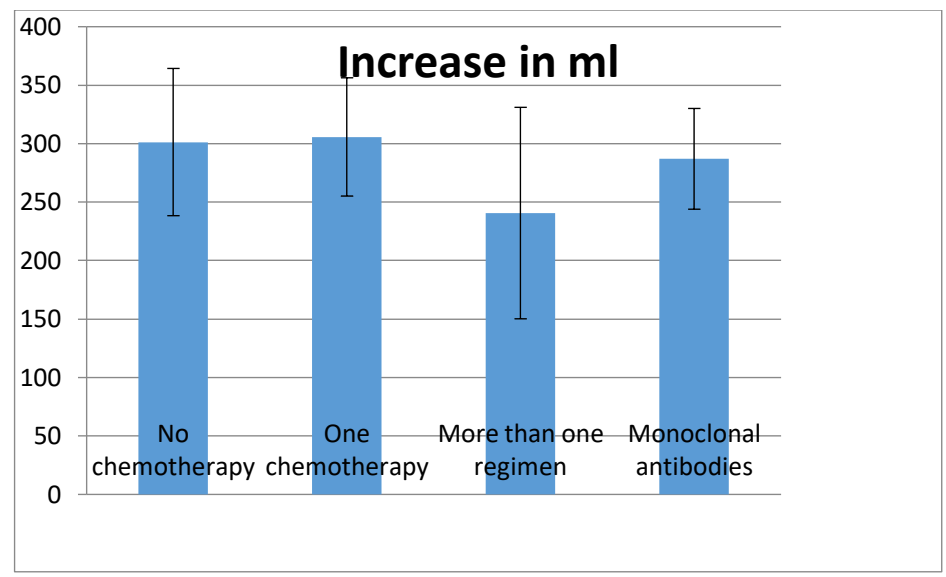

Figure 2. Volume increase in the FLR expressed in milliliters (ml). The FLR increased $301 \mathrm{ml}(95 \mathrm{Cl}$ 238-364) for the group not treated with chemotherapy, $306 \mathrm{ml}(95 \mathrm{Cl} 255-356)$ for the group treated with one regimen of chemotherapy, $241 \mathrm{ml}(95 \mathrm{Cl} 150-331)$ for the group treated with more than one regimen of chemotherapy and 287 (95 Cl 244-330) in the group treated with monoclonal antibodies (Study 1).

The volume increase expressed as the KGR was 18 in the group treated with 1 regimen and lowest in the group treated with more than 1 regimen, with a KGR of 14 . The KGR was 16 in the group treated with no preoperative chemotherapy and in the group treated with monoclonal antibodies (Figure 3). 


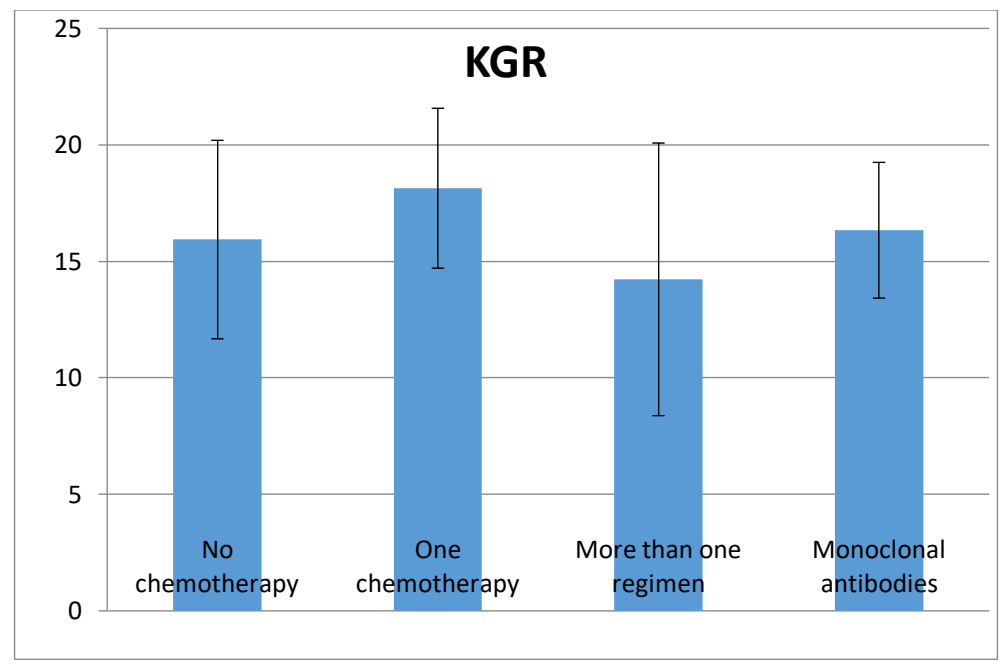

Figure 3. Volume increase in the FLR expressed as the KGR. The KGR was $16(95 \mathrm{Cl} 12-20)$ for the group not treated with chemotherapy, 18 (95 Cl 15-22) for the group treated with one regimen of chemotherapy, 14 (95 $\mathrm{Cl} 8$-20) for the group treated with more than one regimen of chemotherapy and 16 (95 $\mathrm{Cl} 13-19)$ in the group treated with monoclonal antibodies (Study 1).

Neither of the techniques to estimate the volume increase showed any significant difference.

When the volume increase was estimated as a percent or expressed as the KGR, there was no significant difference for irinotecan- or oxaliplatin-based chemotherapy. Patients treated with irinotecan-based chemotherapy had a significantly larger volume increase than those treated with oxaliplatin-based chemotherapy, as expressed in $\mathrm{ml}$.

In the group treated with monoclonal antibodies in addition to chemotherapy, no difference was demonstrated, regardless of whether the volume increase was estimated as a percent, in $\mathrm{ml}$ or expressed as the KGR. 


\section{Study 2}

Forty-eight patients were analyzed in the study due to the exclusion of two patients. One was excluded due to too advanced disease at inclusion, and one was excluded due to missing data. Thirty-four patients (71 \%) underwent PVE, and 14 (29\%) patients underwent PVL, and they were analyzed together. The clinical data and preoperative variables are presented in table 3.

\begin{tabular}{|l|l|}
\hline Number of patients & $\mathbf{4 8}$ \\
\hline Male/female & $36 / 12$ \\
\hline Age (mean, \pm 1 SD) & $65 \pm 12$ \\
\hline BMI (mean, \pm 1 SD) & $26 \pm 4$ \\
\hline ASA (median, range) & $2(1-3)$ \\
\hline ECOG (median, range) & $0(0-1)$ \\
\hline Cortisone treatment & $1(2 \%)$ \\
\hline Diabetes mellitus & $6(13 \%)$ \\
\hline Primary tumor resected/not resected & $30 / 18$ \\
\hline Chemotherapy cycles (mean, \pm 1 SD) & $7 \pm 4$ \\
\hline Response to chemotherapy/stable disease & $37 / 9 *$ \\
\hline $\begin{array}{l}\text { Time (days) between last dose of } \\
\text { chemotherapy and first intervention (median, } \\
\text { range) }\end{array}$ & $35(7-412)$ \\
\hline $\begin{array}{l}\text { Liver metastases at time of surgery (median, } \\
\text { range) }\end{array}$ & $8(1-23)$ \\
\hline $\begin{array}{l}\text { Size (mm) of the largest liver metastasis } \\
\text { (mean, } \pm 1 \text { SD) }\end{array}$ & $48 \pm 38$ \\
\hline Metastases in the FLR & $30(63 \%)$ \\
\hline Extra hepatic disease & $7(15 \%)$ \\
\hline Local lymph node enlargement & $2(4 \%)$ \\
\hline Complications after PVO & $8(17 \%)^{* *}$ \\
\hline
\end{tabular}

Table 3. Clinical variables and preoperative data (study 2 ). ${ }^{*}$ One patient did not receive chemotherapy, and for one patient, the response to the given chemotherapy was difficult to evaluate. ** Data were lacking for one patient. Complications were grade 1 or 2 according to the Clavien-Dindo classification.

For the entire study population, the preoperative sFRL was $21 \%( \pm 5)$. The KGR during the time until either radical hepatectomy or exclusion, which in median was 4 weeks, was $2.8( \pm 2)$. During the first week after PVO, the KGR was $5.4( \pm 4)$, compared to a KGR of $1.5( \pm 2)$ between the first and second volume evaluations $(p<0.05)$. Thirty patients $(63 \%)$ underwent the first volume evaluation within 1 week after PVO, and 13 (27\%) patients underwent the first volume evaluation between 8 and 15 
days after PVO. The second volume evaluation was performed for 23 patients ( $48 \%$ ) within 3 weeks after the first, and 11 (23\%) underwent the second volume evaluation after more than 3 weeks (Figure 4).

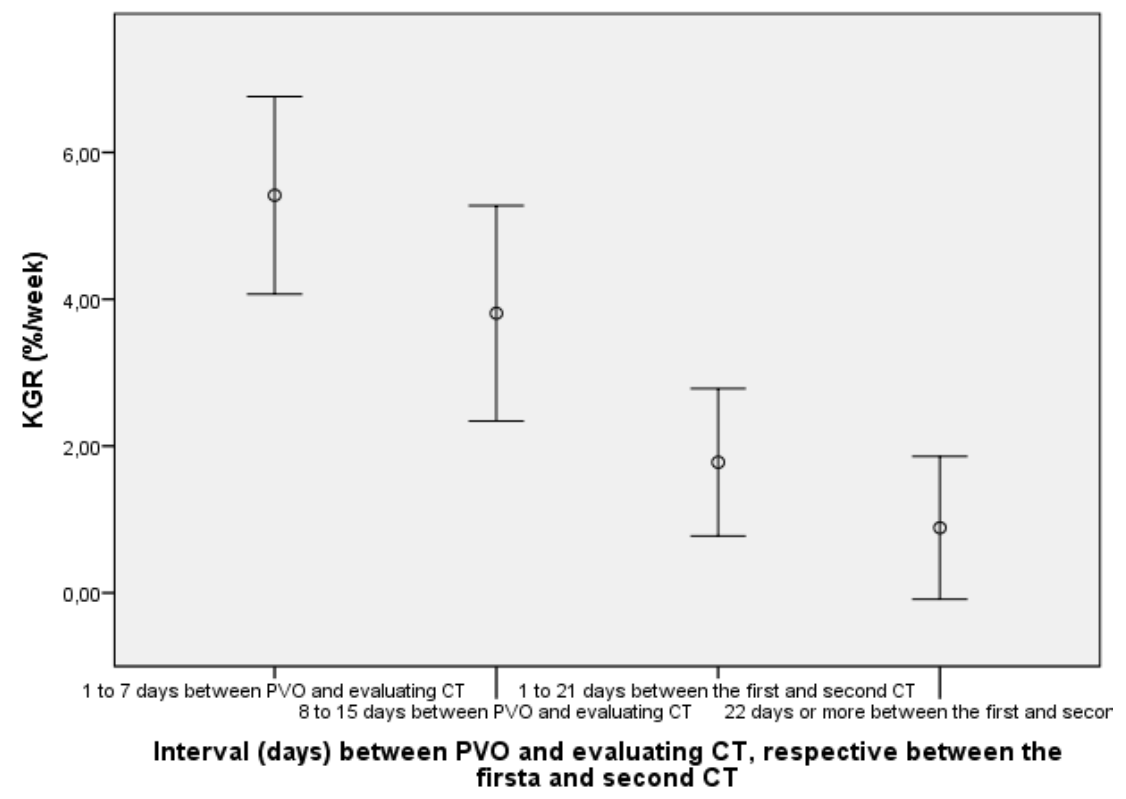

Error Bars: $95 \% \mathrm{Cl}$

Figure 4. Temporal course of the increase in the FLR, with intervals of one to seven days between stage 1/PVE and the first CT and one to twenty-one or twenty-two days or more between the first and second CT. The KGR was $5.4( \pm 4), 3.8( \pm 2), 1.8( \pm 2)$ and $0.9( \pm 1)$, respectively. The differences between the KGR for the interval of one to seven days and those for the intervals of one to twentyone days and twenty-two days or more between the first and second CT evaluations were statistically significant at $p<0.005$ (Study 2).

The KGR values in patients proceeding to radical hepatectomy and in those with insufficient volume increase were $3.4( \pm 2)$ and $1.5( \pm 1)$, respectively $(p=0.015)$. During the first week after PVO, the KGR for the patients who did proceed to radical hepatectomy was $7( \pm 4)$, compared to $4.3( \pm 2)$ for those who failed to reach sufficient volume. However, the difference did not reach statistical significance 
$(p=0.4)$. During the intervals between the first and second volume evaluations, the KGR was $2.2( \pm 2)$ and $0.1( \pm 0.9)$, respectively, $p=0.017$.

Of the patients who did proceed to radical hepatectomy, $29 \%$ reached a sufficient volume of the FLR within the first week. Additionally, $18 \%$ reached a sufficient volume within 15 days, and $7 \%$ reached a sufficient volume within one month from PVO.

Those patients who reached a sufficient volume of the FLR to proceed to radical hepatectomy had a larger prePVO volume of the FLR than those who failed to reach sufficient volume. The SFRL was 23 $\% \pm 4$ compared to $17 \% \pm 5$, but the difference did not reach statistical significance. When analyzing factors that could potentially affect the volume increase, no significant differences could be found, such as differences in prePVO bilirubin levels or BMI. There was no difference in the number of cycles of preoperative chemotherapy.

Radical hepatectomy was performed after 6 (4-14) weeks, and the resection rate was $56 \%$. 


\section{Study 3}

In the study, 48 patients randomized to ALPPS were included, and 49 were randomized to TSH.

Regarding baseline characteristics and preoperative data, there was no significant difference between the groups, and the data are presented in table 4 . There were no differences in postoperative complications, including 90-day mortality. Four and 3 patients died within 90 days of intervention 2 with ALPPS or TSH, respectively. 


\begin{tabular}{|c|c|c|c|}
\hline & ALPPS ( $n=48)$ & TSH $(n=49)$ & $p$-value \\
\hline $\begin{array}{l}\text { Age (years) at the diagnosis of } \\
\text { CRLM }\end{array}$ & $64 \pm 9$ & $63 \pm 12$ & 0.68 \\
\hline Sex (male/female) & $32 / 16$ & $36 / 13$ & 0.46 \\
\hline ASA $1 / 2 / 3$ & $12 / 32 / 4$ & $12 / 28 / 9$ & 0.34 \\
\hline ECOG 0/1/2 & $28 / 17 / 3$ & $30 / 19 / 0$ & 0.20 \\
\hline Synchronous/metachronous & $38 / 10$ & $44 / 5$ & 0.148 \\
\hline $\begin{array}{l}\text { Primary tumor rectum/right } \\
\text { colon/left colon }\end{array}$ & $16 / 11 / 16$ & $14 / 10 / 18$ & 0.729 \\
\hline Number of liver metastases & $8 \pm 4$ & $8 \pm 5$ & 0.48 \\
\hline $\begin{array}{l}\text { Size of the largest liver } \\
\text { metastasis }(\mathrm{mm})\end{array}$ & $56 \pm 42$ & $49 \pm 39$ & 0.405 \\
\hline $\begin{array}{l}\text { Number of patients with } \\
\text { metastases in the FLR on } \\
\text { preoperative } \mathrm{CT} / \mathrm{MRI}\end{array}$ & $29(60 \%)$ & $30(63 \%)$ & 0.84 \\
\hline $\begin{array}{l}\text { Number of metastases in the } \\
\text { FLR }\end{array}$ & $2 \pm 1$ & $3 \pm 2$ & 0.36 \\
\hline $\begin{array}{l}\text { Primary tumor resected at } \\
\text { inclusion (yes/no) }\end{array}$ & $29 / 19$ & $31 / 18$ & 0.77 \\
\hline $\begin{array}{l}\text { CEA level ( } \mathrm{ng} / \mathrm{ml}) \text {, before } \\
\text { preoperative chemotherapy }\end{array}$ & $81 \pm 156$ & $190 \pm 540$ & 0.226 \\
\hline EHD & $11^{*}$ & 7 & 0.59 \\
\hline $\begin{array}{l}\text { Number of cycles of } \\
\text { preoperative chemotherapy }\end{array}$ & $6 \pm 4$ & $7 \pm 4$ & 0.20 \\
\hline $\begin{array}{l}\text { Response to chemotherapy } \\
\text { (stable disease/regression) }\end{array}$ & $9 / 38$ & $10 / 38$ & 0.80 \\
\hline FOLFIRI & 10 & 8 & \\
\hline 5-FU & 1 & 2 & \\
\hline FOLFOX & 19 & 20 & \\
\hline XELOX & 1 & 2 & \\
\hline FOLFOX $+a b$ & 3 & 4 & \\
\hline$X E L O X+a b$ & 3 & 3 & \\
\hline FOLFIRI $+a b$ & 10 & 8 & \\
\hline Xeloda $+a b$ & 0 & 1 & \\
\hline
\end{tabular}

Table 4. Clinical and preoperative data (study 3 and study 4). *Including two patients who had lung metastases resected prior to inclusion in the trial. ab=monoclonal antibody.

Follow-up for all included patients after intervention 1 was 38 months ( $95 \% \mathrm{Cl} 34-42$ ). At the end of follow-up, 27 patients (56\%) randomized to ALPPS were alive, compared to 16 (33\%) of those randomized to TSH ( $p=0.025$ ). Patients who died during follow-up all had either recurrent or residual tumors. 
Analyses of all included patients, according to the intention-to-treat principle (ITT), revealed that the resection rate for patients randomized to ALPPS was $92 \%$, compared to $80 \%$ for patients randomized to TSH ( $p=0.091)$. Significantly more patients randomized to ALPPS than to TSH were assessed as tumor free in the liver at the first postoperative time point: $37(77 \%)$ and $28(57 \%)$, respectively $(p=0.028)$. The median survival values were 46 and 26 months, respectively $(p=0.028)$. Subgroup analysis of the patients randomized to TSH and who received resection with TSH and of those randomized to TSH but treated with rescue ALPPS revealed that 27 patients received resection with TSH, and 12 received rescue ALPPS. The survival values were 35 months and 27 months, respectively $(p=0.076)$.

For patients not proceeding to intervention 2, no difference in survival could be found with regard to group allocation. The median survival for nonresected patients was 13 months (2-24). See figure 5 for the estimated median survival values for patients who received resection with ALPPS, TSH, or rescue ALPPS and for those who did not undergo resection in both groups. 


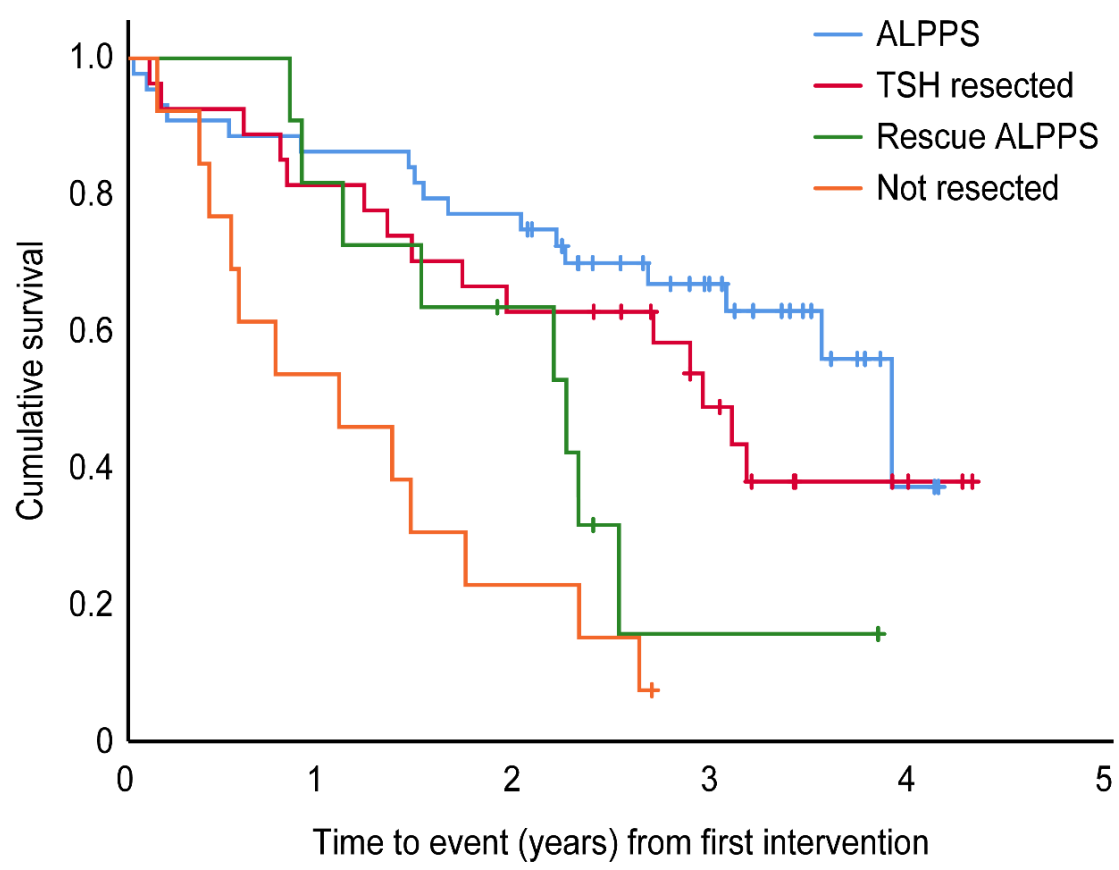

Number at risk:

\begin{tabular}{|c|c|c|c|c|}
\hline ALPPS 44 & 38 & 33 & 18 & 2 \\
\hline TSH 27 & 22 & 17 & 9 & 2 \\
\hline $\begin{array}{l}\text { Rescue } \\
\text { ALPPS }\end{array}$ & 9 & 6 & 1 & 0 \\
\hline lot resected 13 & 7 & 3 & 0 & 0 \\
\hline
\end{tabular}

Figure 5. Estimated median survival for patients who received resection with ALPPS, TSH, or rescue ALPPS and for those who did not receive resection in both groups. The survival values were 46 and 32 months for patients who received resection with ALPPS or TSH, respectively, 27 months for those treated with rescue ALPPS, and 13 months for those who did not receive resection $(95 \% \mathrm{Cl} 36-54$, 29-41, and 14-39 respectively 2-24) (Study 3).

The disease-free survival (DFS) for patients allocated to ALPPS was 11 (95\% Cl 9-12) months, and the DFS for patients randomized to TSH was $8(95 \% \mathrm{Cl} 4-13)$ months $(\mathrm{p}=0.43)$. The most common site for recurrent disease was in the liver for patients randomized to ALPPS and in multiple sites for patients randomized to TSH $(p=0.224)$. The median time to recurrence for patients assessed as tumor free, 
either after radical hepatectomy or after resection of the primary tumor, was 18 months in both groups $(\mathrm{p}=0.89)$.

Regarding postoperative chemotherapy, 19 (40\%) patients randomized to ALPPS and 24 (49\%) patients randomized to TSH received postoperative oncological therapy. The most common agent in both groups was a fluorouracil-based treatment, which 18 (38 respective $37 \%$ ) patients in both groups received $(p=0.209)$. For those patients who completed both interventions, fluorouracil-based treatment was also the most common agent, administered to 16 (33\%) and 19 (39\%) patients, respectively.

\section{Univariable and Multivariable Survival Analyses}

Univariable analysis was performed, and the following factors had a significant impact on outcome. Allocation to ALPPS was associated with improved outcome, as was resection of liver metastases, lower age, small size of the largest metastasis, absence of postoperative complications $\geq 3 a$ according to the Clavien-Dindo classification and being assessed as tumor free in the liver at the first postoperative time point.

On multivariable analysis, the following factors remained significantly associated with improved outcome, allocation to ALPPS, resection of liver metastases, small size of the largest liver metastasis and absence of postoperative complications $\geq 3$ a. A lower ASA classification was also significantly associated with improved outcome in the multivariable analysis (table 5). 


\begin{tabular}{|c|c|c|c|c|c|c|c|c|}
\hline \multirow[b]{2}{*}{ Covariate } & \multicolumn{4}{|c|}{ Univariable analysis } & \multicolumn{4}{|c|}{ Multivariable analysis* } \\
\hline & Coefficient & $\mathrm{HR}$ & $95 \% \mathrm{Cl}$ & $\begin{array}{l}p- \\
\text { value }\end{array}$ & Coefficient & $H R$ & $\begin{array}{l}95 \% \\
\mathrm{Cl}\end{array}$ & $\begin{array}{l}\mathrm{p} \text { - } \\
\text { value }\end{array}$ \\
\hline $\begin{array}{l}\text { Allocated } \\
\text { treatment } \\
\text { (ALPPS/TSH) }\end{array}$ & -0.656 & 0.519 & $\begin{array}{l}0.3- \\
0.91\end{array}$ & 0.022 & -0.908 & 0.403 & $\begin{array}{l}0.18- \\
0.9\end{array}$ & 0.026 \\
\hline $\begin{array}{l}\text { Response to } \\
\text { chemotherapy } \\
\text { (regression/stable) }\end{array}$ & -0.32 & 0.72 & $\begin{array}{l}0.38- \\
1.4\end{array}$ & 0.335 & & & & \\
\hline $\begin{array}{l}\text { Number of cycles } \\
\text { of preoperative } \\
\text { chemotherapy }\end{array}$ & -0.004 & 1 & $\begin{array}{l}0.92- \\
1.1\end{array}$ & 0.915 & & & & \\
\hline No EHD & -0.375 & 0.69 & $\begin{array}{l}0.36- \\
1.31\end{array}$ & 0.257 & & & & \\
\hline $\begin{array}{l}\text { Liver metastases } \\
\text { not resected }\end{array}$ & 1.29 & 3.64 & $\begin{array}{l}1.82- \\
7.29\end{array}$ & $<0.001$ & 1.782 & 5.94 & $\begin{array}{l}1.63- \\
21.64 \\
\end{array}$ & 0.007 \\
\hline $\begin{array}{l}\text { Age (decades) at } \\
\text { diagnosis of liver } \\
\text { metastases }\end{array}$ & 0.329 & 1.39 & $\begin{array}{l}1.02- \\
1.9\end{array}$ & 0.035 & & & & \\
\hline ASA & 0.41 & 1.51 & $\begin{array}{l}0.96- \\
2.39\end{array}$ & 0.078 & 1.121 & 3.1 & $\begin{array}{l}1.4- \\
6.8 \\
\end{array}$ & 0.006 \\
\hline ECOG & 0.209 & 1.23 & $\begin{array}{l}0.79- \\
1.93\end{array}$ & 0.36 & & & & \\
\hline $\begin{array}{l}\text { Size }(\mathrm{cm}) \text { of the } \\
\text { largest liver } \\
\text { metastases }\end{array}$ & 0.12 & 1.13 & $1.1-1.2$ & $<0.001$ & 0.179 & 1.2 & $\begin{array}{l}1.1- \\
1.3\end{array}$ & $<0.001$ \\
\hline $\begin{array}{l}\text { Number of liver } \\
\text { metastases }\end{array}$ & 0.037 & 1.04 & $\begin{array}{l}0.99- \\
1.09\end{array}$ & 0.152 & & & & \\
\hline $\begin{array}{l}\text { Time (weeks) } \\
\text { between } \\
\text { intervention } 1 \text { and } \\
\text { intervention } 2\end{array}$ & -0.021 & 0.979 & $\begin{array}{l}0.93- \\
1.04\end{array}$ & 0.474 & & & & \\
\hline $\begin{array}{l}\text { Postoperative } \\
\text { complications } \geq \\
3 a^{* *}\end{array}$ & 1.26 & 3.54 & $\begin{array}{l}1.65- \\
7.59\end{array}$ & 0.001 & 1.244 & 3.47 & $\begin{array}{l}0.12- \\
0.69\end{array}$ & 0.005 \\
\hline $\begin{array}{l}\text { Tumor free in the } \\
\text { liver at first } \\
\text { postoperative } \\
\text { follow-up }\end{array}$ & -1.12 & 0.30 & $\begin{array}{l}0.16- \\
0.56\end{array}$ & $<0.001$ & & & & \\
\hline
\end{tabular}

Table 5. Univariable and multivariable Cox regression analyses (study 3). *Only variables with $p \leq 0.05$ are reported ${ }^{* *}$ Highest grade of complication after intervention 1 or intervention 2 


\section{Study 4}

In the analysis, 48 patients randomized to ALPPS were included, and 49 were randomized to TSH. No significant difference was found regarding clinical and preoperative data (table 4).

\section{Summary of costs and resource use during the entire study period}

During the entire study period, the mean cost for patients randomized to ALPPS was 77,530 EUR (95 $\% 58,602-96,459)$ compared to 64,868 EUR $(95 \% \mathrm{Cl} 50,654-79,082)$ for those randomized to TSH $(p=0.283)$. See table 6 for costs during periods 1-3. 


\begin{tabular}{|c|c|c|c|c|}
\hline & ALPPS & TSH & Mean difference & P-value \\
\hline \multicolumn{5}{|l|}{ Period 1} \\
\hline Intervention 1, number of patients & 48 & 49 & & \\
\hline $\begin{array}{l}\text { Cost, surgical procedure and care at } \\
\text { the recovery ward }\end{array}$ & $7,714(7,501-7,926)$ & $7,566(6906-8226)$ & 148 & 0.671 \\
\hline Total cost of intervention 1 & $11,685(11,305-12,064)$ & $12,269(10,981-13,557)$ & -584 & 0.388 \\
\hline Intervention 2 , number of patients & $45^{*}$ & $40 * *$ & & \\
\hline $\begin{array}{l}\text { Cost, surgical procedure and care at } \\
\text { the recovery ward }\end{array}$ & $7,114(6,830-7,397)$ & $9,882(8,799-10,966)$ & $-2,769$ & $<0.001$ \\
\hline Total cost of intervention 2 & $17,973(16,015-19,930)$ & $22,367(17,266-27,467)$ & $-4,394$ & 0.096 \\
\hline $\begin{array}{l}\text { Total cost of interventions } 1 \text { and } \\
2^{* * *}\end{array}$ & $29,017(26,729-31,305)$ & $31,388(26,238-36,538)$ & $-2,371$ & 0.403 \\
\hline $\begin{array}{l}\text { Postoperative complications } 3 a-3 b \text {, } \\
\text { number of patients }\end{array}$ & 16 & 15 & & \\
\hline Cost, postoperative complications & $1,480(653-2,307)$ & $797(220-1,373)$ & 684 & 0.163 \\
\hline $\begin{array}{l}\text { Care at the intensive care unit, } \\
\text { number of patients }\end{array}$ & 5 & 5 & & \\
\hline Cost, intensive care unit & 18,207 & 18,207 & 0 & \\
\hline Readmitted, number of patients & 10 & 12 & & \\
\hline Cost, readmitted & $9,388(3,554-15,223)$ & $5,835(4,182-7,487)$ & 3554 & 0.168 \\
\hline Total cost of period 1 & $54,311(36,224-72,398)$ & $50,222(38,510-61,935)$ & 4089 & 0.702 \\
\hline \multicolumn{5}{|l|}{ Period 2} \\
\hline Number of patients" & 47 & 48 & & \\
\hline Resection of primary tumor $(13 / 10)$ & $8,202(7,776-8,629)$ & $8,527(8,057-8,997)$ & -325 & 0.273 \\
\hline Resection EHD (8/9) & $9,715(8,357-11,073)$ & $9,701(6,601-12,796)$ & 14 & 0.993 \\
\hline Diagnostic procedure $(42 / 38)$ & $1,806(1,653-1,959)$ & $1,405(1,214-1,596)$ & 401 & 0.001 \\
\hline Chemotherapy (28/27) & $18,431(9,811-27,051)$ & $10,922(4,269-17,575)$ & 7,509 & 0.165 \\
\hline Total cost of period $2^{\text {ๆๆ }}$ & $18,053(11,950-24,156)$ & $12,114(7,247-16,980)$ & 5,940 & 0.128 \\
\hline \multicolumn{5}{|l|}{ Period 3} \\
\hline Number of patients" & 40 & 36 & & 0.325 \\
\hline Resection EHD (4/4) & $7,284(4,080-10,489)$ & $10,287(3,110-17,463)$ & $-3,002$ & 0.270 \\
\hline Diagnostic procedure $(32 / 27)$ & $982(817-1,146)$ & $1,201(1,021-1,382)$ & -220 & 0.071 \\
\hline Chemotherapy (10/5) & $27,768(13,679-41,857)$ & $24,637(585-48,690)$ & 3,131 & 0.775 \\
\hline Total cost of period 3 & $10,249(4,284-16,215)$ & $7,028(4,886-12,655)$ & 3,222 & 0.413 \\
\hline Total cost of periods 1-3 & $77,530(58,602-96,459)$ & $64,868(50,654-79,082)$ & 12,662 & 0.283 \\
\hline $\begin{array}{l}\text { Number of patients alive at the end } \\
\text { of period } 3\end{array}$ & 35 & 23 & & 0.021 \\
\hline
\end{tabular}

Table 6. Included costs (study 4). All costs are expressed as the mean $(95 \% \mathrm{Cl})$ and in euros. * Including one patient resected in violation of the protocol. ${ }^{* *}$ Including 12 patients who crossed over to rescue ALPPS and one patient who had carcinomatosis discovered during explorative laparotomy. ***Including the costs for the surgical procedures, care at the recovery ward, and care at the surgical ward and the cost for the microscopic histopathological examination. "Number of patients alive at approximately 4 weeks after discharge after the last intervention, including patients not proceeding to intervention $2{ }^{19}$ Excluding the costs for intervention 1 and intervention 2 and the first 30 days after discharge from intervention $2{ }^{\text {กा9 } N u m b e r}$ of patients alive at the start of periods 2 and 3 , respectively. 


\section{Cost and resource use for period one}

All included patients underwent intervention 1, which consisted of ALPPS intervention 1 for patients randomized to ALPPS and PVE or PVL for patients randomized to TSH. The mean cost of intervention 1 did not differ between the groups. The mean cost for the subgroup of patients treated with PVE was higher than that for the patients treated with ALPPS intervention 1. In addition, some patients treated with PVE had metastases in the FLR resected, which further increased the cost. If the costs of length of stay and radiological exams before intervention 2 were included, there was no significant difference in costs $(p=0.388)$.

Forty-four patients randomized to ALPPS underwent intervention 2, and one patient underwent intervention 2 in violation of the protocol but was included in the cost analysis. Forty patients randomized to TSH underwent intervention 2, including 12 patients treated with rescue ALPPS and 1 patient who only underwent exploratory laparotomy due to the finding of carcinomatosis, and hence, no resection was performed. The cost for the surgical procedures of intervention 2 was significantly higher for patients randomized to TSH than for patients randomized to ALPPS $(p<0.001)$. The total cost of intervention 2, including length of stay, was still higher for patients randomized to TSH than for those randomized to ALPPS, but the difference did not reach statistical significance $(p=0.096)$.

The total cost of intervention 1 and intervention 2 did not differ between patients randomized to ALPPS or TSH $(p=0.403)$.

\section{Cost and resource use during period two}

Forty-seven patients randomized to ALPPS and 48 patients randomized to TSH were alive at the beginning of period 2 . 
Nineteen ( $40 \%$ ) of the patients randomized to ALPPS and $18(37 \%)$ of the patients randomized to TSH had the primary tumor still in situ at the beginning of period 2. During this time period, 13 (27 $\%)$ and 10 (20\%) had the primary tumor resected.

During period 2, 28 (58\%) patients randomized to ALPPS and 27 (56\%) randomized to TSH were treated with chemotherapy, and the difference in costs for chemotherapy did not differ.

Eight patients randomized to ALPPS were treated for recurrent disease with either resection or ablation, compared to 2 patients randomized to TSH, with no significant difference in associated costs $(p=0.218)$.

During period 2, there was no significant difference in cost and resource use between patients randomized to ALPPS compared to TSH $(p=0.128)$.

\section{Cost and resource use during period three}

Forty patients randomized to ALPPS and 36 (73\%) patients randomized to TSH were alive at the beginning of period $3(p=0.325)$. Of those, 9 patients and 23 patients $(25 \%)$ were assessed as tumor free in the ALPPS and TSH groups, respectively, $p=0.665$.

No patient had the primary tumor resected after period 2. Four (13\%) patients randomized to ALPPS had recurrent tumor in the liver and underwent resection or ablation, compared to 4 (15\%) patients randomized to TSH $(p=0.80)$. Ten $(25 \%)$ and $5(14 \%)$ patients, respectively, received chemotherapy during period $3(p=0.775)$.

During period 3, the costs for patients randomized to ALPPS were higher than the costs for patients randomized to $\mathrm{TSH}$, but the difference did not reach statistical significance $(\mathrm{p}=0.413)$. 


\section{Health outcomes}

The response rate was highest in both groups before intervention 1 and was for patients randomized to ALPPS $85 \%$ and $90 \%$ for patients randomized to TSH. The response rate declined during the study period and was lowest at 24 months.

There was no significant difference in mean quality-adjusted survival, estimated with the EQ-5D, for patients randomized to ALPPS or TSH (1.25 (95 \% Cl 1.08-1.42) and 1.11 (95 \% Cl 0.94-1.27), respectively, $p=0.234)$. There was no difference in life years for patients randomized to ALPPS and TSH (1.68 (95 \% Cl 1.5-1.87) and $1.55(95 \% \mathrm{Cl} 1.37-1.73)$, respectively, $\mathrm{p}=0.314)$.

\section{Cost effectiveness}

The mean cost difference between ALPPS and TSH was 12,662 EUR (95 \% Cl -10,728-36,051). The mean difference in QALYs was $0.1285(95 \% \mathrm{Cl}-0.11-0.36 ; p=0.28)$, and the mean difference in LYs was $0.1296(95 \% \mathrm{Cl}-0.12-0.38, \mathrm{p}=0.314)$. The ICER was therefore 93,186 for QALYs and 92,414 for LYs.

The cost effectiveness analysis revealed that the mean cost difference between ALPPS and TSH was $12,662 €(95 \% \mathrm{Cl}-10,728-36,051, \mathrm{p}=0.283)$. The mean difference in life years was $0.1296(95 \% \mathrm{Cl}-$ 0.12-0.38, $\mathrm{p}=0.314)$, and the mean difference in QALYs was $0.1285(95 \% \mathrm{Cl}-0.11-0.36, \mathrm{p}=0.28)$. Accordingly, the ICER for life years was 92,414, and the ICER for QALYs was 93,186.

The joint distributions of costs and outcomes, expressed as QALYs and LYs, are shown in figures 6 and 7. 


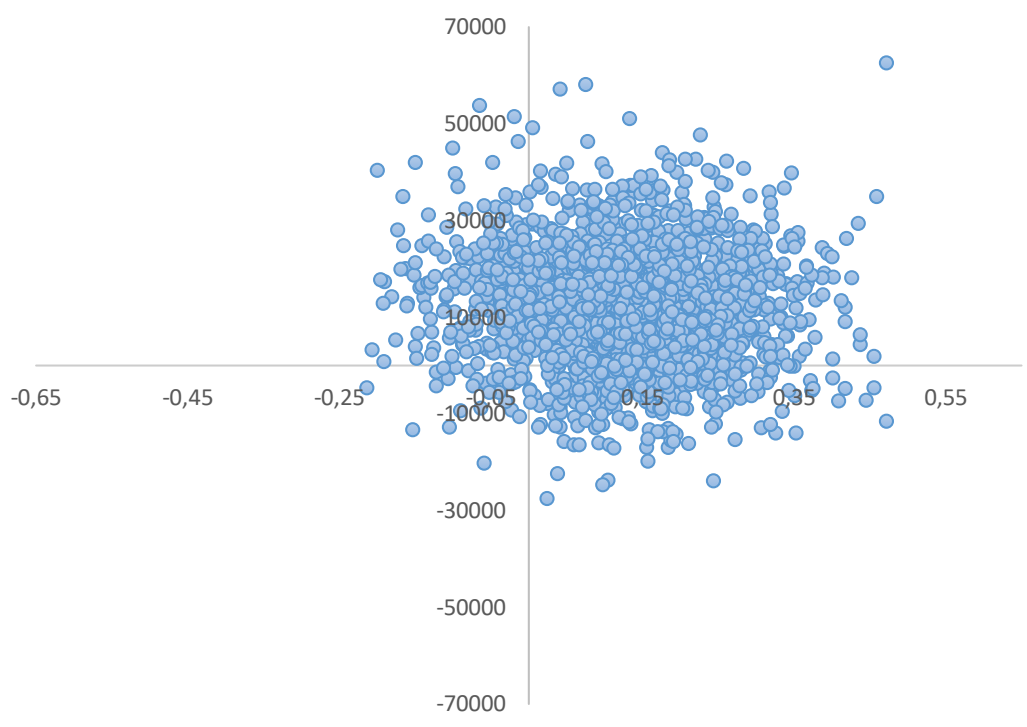

Figure 6. Incremental cost and QALYs for ALPPS compared to TSH. Result from the probabilistic analysis on a cost-effectiveness plane. Incremental cost and effect, expressed as QALYs, are calculated as ALPPS minus TSH. 


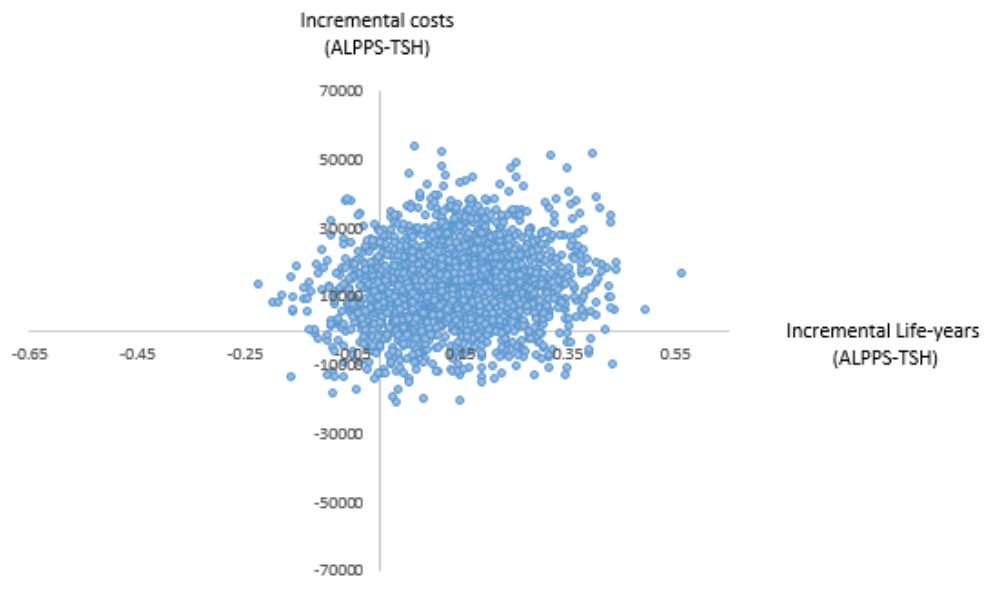

Figure 7. Incremental cost and life years for ALPPS compared to TSH. Result from the probabilistic analysis on a cost-effectiveness plane. Incremental cost and effect, expressed as life years, are calculated as ALPPS minus TSH. 


\section{Discussion}

The focus of this thesis was to study different aspects of ALPPS and PVO in patients with advanced CRLM. Those patients receiving such treatments represent a rather small proportion of patients with CRLM undergoing resection. Due to the rapid development of surgical techniques and oncological treatment, the number of such patients might increase. It is therefore important to study the different aspects of the treatments as well as the outcomes, including outcomes related to survival but also related to health-related quality of life and the health economic outcomes.

Study 1 is so far the largest study conducted on patients undergoing ALPPS and treated with preoperative chemotherapy; no negative impact on the volume increase of the FLR could be found.

Patients who are considered for ALPPS have advanced disease. They are often assessed as borderline resectable or even unresectable without conversion chemotherapy. For this category of patients with advanced CRLM, the resection rate increases if chemotherapy is administered preoperatively [50]. The outcome, in terms of progression-free survival (PFS) and overall survival (OS), is also improved [44]. Furthermore, preoperative chemotherapy may provide some indication of tumor biology. Patients with progressive disease on the first line of chemotherapy may have a more aggressive disease and may therefore not benefit from undergoing the ALPPS procedure. This has previously been shown to be a risk factor for not proceeding to the second interventions for patients undergoing TSH [17] and is most likely also applicable for patients who are considered for ALPPS.

One previous concern has been that preoperative chemotherapy may negatively affect the volume increase. The subject has previously not been well studied for patients undergoing ALPPS. In one study performed on patients with other diagnoses as well as CRLM, it was found that the volume increase was lower for those treated with chemotherapy [196]. However, the volume increase was sufficient to proceed to intervention 2 . 
The effect on volume increase for patients undergoing TSH is more extensively studied. The results have also been conflicting in this setting, and some studies have shown an impaired volume increase, especially with longer preoperative chemotherapy [197, 198]. Other studies could not find a difference in volume increase $[68,69]$. The results have also been conflicting for patients treated with monoclonal antibodies in addition to chemotherapy. Some studies found a decreased volume increase [66], and other studies, in contrast, found no negative impact on the volume increase [199].

The exact cellular and molecular mechanism through which chemotherapy and monoclonal antibodies impair the volume increase is not fully understood. Most studies regarding chemotherapy-associated liver injury (CALI) are based on animal models.

Whether steatohepatitis caused by irinotecan impairs volume increases is not fully known, neither if the mechanism would be the same as other causes of steatohepatitis

The regeneration process is complex, and there are therefore many mechanisms that could be affected. One could speculate that the steatohepatitis caused by irinotecan could potentially affect the expression of inflammatory cytokines, such as TNF- $\alpha$ and IL-6, which participate in liver regeneration by regulating the expression of transcription factors $[95,96]$. Oxaliplatin, another commonly used chemotherapy agent for patients with advanced CRLM, may affect the cell cycle by inducing signal pathways that force the cell into the senescent phase, in which the cell does not regenerate [200].

Although some data indicate that preoperative chemotherapy may impair the regeneration process, we could not find any clinical impact on the volume increase in the FRL. There was no significant difference in volume increase between the groups. Furthermore, none of the patients who did not proceed to intervention 2 were unable to because of an insufficient volume increase. Most likely, the regeneration stimulus induced by ALPPS is so large that eventual impairment is not detectable in the clinical setting. This is partly supported by data indicating that the regeneration response in ALPPS is earlier and more pronounced than that in TSH [97-99]. 
There were some limitations to this study. First, it did not explore whether there was any difference in liver function between the groups. Some previous studies indicated that the volume increase preceded the increase in function for patients undergoing ALPPS [170]. However, there were no data that suggested that liver function differed between the groups.

Another limitation is that the proportion of patients who did not receive preoperative chemotherapy was only $10 \%$ of the cohort, which may weaken the statistical analyses.

Finally, there may be a bias in the data in the registry, given that the registry is voluntary and not validated.

However, even with these objectives and limitations, our conclusion is that chemotherapy does not negatively affect the volume increase for patients undergoing ALPPS and that it should be considered for all patients undergoing ALPPS.

In study 2, it was found that the volume increase after PVO was largest in the first week, which has not previously been shown. Furthermore, it was shown that patients with a very low volume of the FLR did not reach a sufficient volume to proceed to radical hepatectomy. Finally, patients with a low KGR at the first week were at risk to not gain sufficient volume of the FLR.

The gradual decline in volume increase was evident in both the group that did proceed to radical hepatectomy and the group that failed to achieve sufficient volume. However, it was more pronounced in the group that did not proceed to radical hepatectomy; during the first week, the KGR was 1.5 , but during the interval between the first and second volume evaluations, it declined to 0.1. The fact that patients with a low KGR are at high risk of failing to achieve sufficient volume of the FLR has previously been shown by Vauthey et al [117], but their study included a longer interval between PVE and radiological evaluation of approximately 1 month. If the first evaluation is performed approximately one week after PVE, it is likely to identify those that will reach sufficient 
volume and may shortly thereafter proceed to radical hepatectomy, as well as those that are at high risk to fail to reach sufficient volume, even with longer waiting time.

In this study, it was also found that patients with a presFLR $\leq 17$ did not reach sufficient volume after PVO. One strategy for such patients may be to consistently also embolize segment 4 . In a previous study comparing patients undergoing right PVE and right + segment 4 PVE [201], it was found that the prePVE volume was significantly lower in the latter group, but the postPVE volume did not differ between the two groups, nor did the resection rate. These findings indicate that with the inclusion of segment 4 branches, the hypertrophy stimulus is more pronounced. Furthermore, no difference in the complication rate could be found, suggesting that the procedure is safe.

One limitation of this study is that liver function was not analyzed. It is therefore not possible to conclude whether there was an underlying difference in liver function that could explain the difference in volume increase. Some data indicate that for patients treated with PVE, liver function increases before the volume increase [138]. It is, however, unlikely that the patients who failed to reach sufficient volume would have had sufficient function to proceed to radical hepatectomy without a significant risk of PHLF given that the volume at the last evaluation was only $21 \%$.

Study 3 was a preplanned study based on data from the first randomized controlled study in which patients with CRLM were randomized to ALPPS or TSH. It was found that patients randomized to ALPPS had a significantly longer survival than patients randomized to TSH: 46 compared to 26 months, respectively.

In the comparison of the patients from the ALPPS and TSH groups that completed the treatments, there was no significant difference, although the survival for patients randomized to ALPPS was still longer and was 46 months, compared to 32 months for patients randomized to TSH. 
The resection rate was higher for patients randomized to ALPPS than for those randomized to TSH, which may be a contributing factor to the improved outcome. That the resection of liver metastases was an important factor in determining the outcome was supported by the regression analysis.

The survival of patients treated with rescue ALPPS was lower than that of patients resected in both the ALPPS group and the TSH group. This may, at least partly, be explained by the long interval from the first intervention until ALPPS intervention 2. The study was not designed to analyze factors that may affect the outcome. One possible explanation may be the longer interval between preoperative and postoperative chemotherapy, since a shorter chemotherapy-free interval may improve the outcome, as previously shown [202]. However, in line with our previous results, one factor that may improve the outcome for this group of patients is an early decision to proceed to rescue ALPPS for those patients treated with PVO and who have an insufficient volume increase of the FLR and a low KGR at the first radiological evaluation.

It has been argued that ALPPS as a surgical procedure should not be applied due to the high rate of postoperative complications [203]. In the LIGRO trial [178], there was no difference in postoperative morbidity and mortality for patients randomized to ALPPS or TSH, and the overall complication rates were comparable with those in previous results [174]. Another argument against the ALPPS procedure has been that its outcome is comparable with the outcome if this category of patients was treated with palliative chemotherapy [177]. However, the survival for the included patients in the LIGRO trial study was longer.

One relatively new technique is LVD (liver venous deprivation). It was initially described as a sequential technique in which PVE was performed first, and after 1-2 weeks, LVD was performed [204]. During the procedure, both the portal and hepatic vein to the lobe to be resected are embolized. The technique has since evolved to be performed as one procedure, with a resection rate from 86 to $100 \%$ and a low frequency of postoperative complications [205-207]. Whether LVD can replace ALPPS remains to be seen, and further studies are warranted. 
An argument for treating patients with advanced CRLM with resection instead of palliative chemotherapy can be made from a health economic perspective. In a previous health economic analysis of patients with CRLM, patients who underwent resection had a higher quality of life than those treated with palliative chemotherapy [189]. Furthermore, resection was superior to palliative chemotherapy in terms of resource use $[191,192]$. However, the study populations in those studies included patients undergoing smaller resections, including wedge resections and segmentectomies, and it is therefore not possible to conclude that this result also applies to patients undergoing ALPPS and TSH.

In the health economic analysis, no significant differences could be found between patients randomized to ALPPS and TSH in terms of QoL, costs or resource use. However, there was a tendency toward a higher cost for patients randomized to ALPPS than for those randomized to TSH, including costs during follow-up. This may reflect that a larger proportion of patients randomized to ALPPS than randomized to TSH were treated with chemotherapy. To draw a conclusion regarding cost effectiveness, further studies beyond 2 years are needed. However, given the improved survival, and at least not inferior cost effectiveness, ALPPS is still a method to consider for patients with advanced CRLM and a low volume of the FLR, although less invasive methods should be explored. 


\section{Conclusion}

In conclusion, the main results from this thesis are that for patients undergoing ALPPS, preoperative chemotherapy does not negatively impact the volume increase of the FLR and should therefore be considered if indicated. Furthermore, for patients undergoing TSH, it is important to perform the first radiological volume evaluation early to detect those at risk for insufficient volume increase and therefore not delay the eventual need for another technique to increase the volume of the FLR. For this group of patients, resection of liver metastases results in improved survival, and the resection rate was higher for patients randomized to ALPPS than for those randomized to TSH. Finally, no significant difference from a health economic perspective could be found between patients randomized to ALPPS or TSH, and neither technique could, based on these results, be determined to be inferior. 


\section{Future perspectives}

Due to advances in liver surgery and oncological therapy in recent decades, the number of patients who are considered for surgery is increasing. There is still a significant proportion of patients who, at the time of diagnosis, have advanced disease. It is encouraging to think that this group of patients will be reduced with increased screening for colorectal cancer. However, it is unlikely, at least within the foreseeable future, that there will be no patients with CRLM or, including those with advanced tumors burden.

Research surrounding less invasive methods to increase the volume of the FLR is expanding. The optimal technique, with a low procedural complication rate, a high technical success rate, and a predicable increase in the volume of the FLR, has yet to be discovered. Perhaps LVD will prove to be that technique, but further research is warranted.

The development of minimally invasive surgery, including robotic surgery, will most likely continue and, to a further degree, such surgeries will be applied in large and technically challenging resections. Minimally invasive surgery has been shown to reduce the rate of postoperative complications [208]. Although major liver surgery today has a relatively low morbidity and mortality rate compared with the surgeries of the past, the rates can still be further decreased.

One of the large challenges is to accurately predict which patients will benefit from liver resection and which are at a high risk of early recurrence. Given the rapid development and understanding of oncogenes, it might be better to estimate the prognosis of each patient and individualize the treatment. This may also lead to further development and individualization of oncological therapy.

Finally, further health economic studies are warranted, both to study the effects of major liver surgery and oncological therapy on quality of life and to ensure that the surgical procedures are economically effective. 


\section{Acknowledgements}

There are many people to whom I am grateful, including the following individuals:

Bergthor Björnsson, my current primary supervisor and former assistant supervisor

Per Sandström, my former primary supervisor and current assistant supervisor

Tommy Sundqvist, former assistant supervisor

Lars Valter, statistician and cowriter in the first study.

I am also thankful to all the collaborators in the LIGRO group. 


\section{References}

1. Ferlay, J., et al., Estimating the global cancer incidence and mortality in 2018: GLOBOCAN sources and methods. Int J Cancer, 2019. 144(8): p. 1941-1953.

2. Leporrier, J., et al., A population-based study of the incidence, management and prognosis of hepatic metastases from colorectal cancer. Br J Surg, 2006. 93(4): p. 465-74.

3. Manfredi, S., et al., Epidemiology and management of liver metastases from colorectal cancer. Ann Surg, 2006. 244(2): p. 254-9.

4. Landreau, P., et al., Incidence and survival in late liver metastases of colorectal cancer. J Gastroenterol Hepatol, 2015. 30(1): p. 82-5.

5. Jones, R.P., et al., Systematic review and meta-analysis of follow-up after hepatectomy for colorectal liver metastases. Br J Surg, 2012. 99(4): p. 477-86.

6. Nordlinger, B., et al., Surgical resection of colorectal carcinoma metastases to the liver. A prognostic scoring system to improve case selection, based on 1568 patients. Association Francaise de Chirurgie. Cancer, 1996. 77(7): p. 1254-62.

7. Fong, Y., et al., Clinical score for predicting recurrence after hepatic resection for metastatic colorectal cancer: analysis of 1001 consecutive cases. Ann Surg, 1999. 230(3): p. 309-18; discussion 318-21.

8. Schreckenbach, T., et al., The clinical relevance of the Fong and the Nordlinger scores in the era of effective neoadjuvant chemotherapy for colorectal liver metastasis. Surg Today, 2015. 45(12): p. 1527-34.

9. Ayez, N., et al., Is the clinical risk score for patients with colorectal liver metastases still useable in the era of effective neoadjuvant chemotherapy? Ann Surg Oncol, 2011. 18(10): p. 2757-63.

10. Wang, X.Y., et al., Meta-analysis of the association between primary tumour location and prognosis after surgical resection of colorectal liver metastases. Br J Surg, 2019. 106(13): p. 1747-1760.

11. Liu, W., et al., The primary tumor location impacts survival outcome of colorectal liver metastases after hepatic resection: A systematic review and meta-analysis. Eur J Surg Oncol, 2019. 45(8): p. 1349-1356.

12. Tsai, M.S., et al., Clinicopathological features and prognosis in resectable synchronous and metachronous colorectal liver metastasis. Ann Surg Oncol, 2007. 14(2): p. 786-94.

13. Engstrand, J., et al., Synchronous and metachronous liver metastases in patients with colorectal cancer-towards a clinically relevant definition. World J Surg Oncol, 2019. 17(1): p. 228.

14. Slesser, A.A., et al., The tumour biology of synchronous and metachronous colorectal liver metastases: a systematic review. Clin Exp Metastasis, 2013. 30(4): p. 457-70.

15. Hokuto, D., et al., The prognosis of liver resection for patients with four or more colorectal liver metastases has not improved in the era of modern chemotherapy. J Surg Oncol, 2016. 114(8): p. 959-965.

16. Vigano, L., et al., Early recurrence after liver resection for colorectal metastases: risk factors, prognosis, and treatment. A LiverMetSurvey-based study of 6,025 patients. Ann Surg Oncol, 2014. 21(4): p. 1276-86.

17. Imai, K., et al., Failure to Achieve a 2-Stage Hepatectomy for Colorectal Liver Metastases: How to Prevent It? Ann Surg, 2015. 262(5): p. 772-8; discussion 778-9.

18. Sasaki, K., et al., The Tumor Burden Score: A New "Metro-ticket" Prognostic Tool For Colorectal Liver Metastases Based on Tumor Size and Number of Tumors. Ann Surg, 2018. 267(1): p. 132-141. 
19. John, S.K., et al., Prognostic factors and survival after resection of colorectal liver metastasis in the era of preoperative chemotherapy: an 11-year single-centre study. Dig Surg, 2013. 30(4-6): p. 293-301.

20. Margonis, G.A., et al., Prognostic Factors Change Over Time After Hepatectomy for Colorectal Liver Metastases: A Multi-institutional, International Analysis of 1099 Patients. Ann Surg, 2019. 269(6): p. 1129-1137.

21. Sadot, E., et al., Resection margin and survival in 2368 patients undergoing hepatic resection for metastatic colorectal cancer: surgical technique or biologic surrogate? Ann Surg, 2015. 262(3): p. 476-85; discussion 483-5.

22. Hamady, Z.Z., et al., One-millimeter cancer-free margin is curative for colorectal liver metastases: a propensity score case-match approach. Ann Surg, 2014. 259(3): p. 543-8.

23. Memeo, R., et al., Margin Status is Still an Important Prognostic Factor in Hepatectomies for Colorectal Liver Metastases: A Propensity Score Matching Analysis. World J Surg, 2018. 42(3): p. 892-901.

24. Martin, E.W., Jr., et al., The use of CEA as an early indicator for gastrointestinal tumor recurrence and second-look procedures. Cancer, 1977. 39(2): p. 440-6.

25. Sasaki, K., et al., Pre-hepatectomy carcinoembryonic antigen (CEA) levels among patients undergoing resection of colorectal liver metastases: do CEA levels still have prognostic implications? HPB (Oxford), 2016. 18(12): p. 1000-1009.

26. Vauthey, J.N., et al., RAS mutation status predicts survival and patterns of recurrence in patients undergoing hepatectomy for colorectal liver metastases. Ann Surg, 2013. 258(4): p. 619-26; discussion 626-7.

27. Margonis, G.A., et al., Codon 13 KRAS mutation predicts patterns of recurrence in patients undergoing hepatectomy for colorectal liver metastases. Cancer, 2016. 122(17): p. 2698-707.

28. Karagkounis, G., et al., Incidence and prognostic impact of KRAS and BRAF mutation in patients undergoing liver surgery for colorectal metastases. Cancer, 2013. 119(23): p. 413744.

29. Brudvik, K.W., et al., RAS Mutation Clinical Risk Score to Predict Survival After Resection of Colorectal Liver Metastases. Ann Surg, 2019. 269(1): p. 120-126.

30. Nystrom, H., et al., Liver-metastatic potential of colorectal cancer is related to the stromal composition of the tumour. Anticancer Res, 2012. 32(12): p. 5183-91.

31. Dupre, A., et al., Validation of clinical prognostic scores for patients treated with curativeintent for recurrent colorectal liver metastases. J Surg Oncol, 2018. 117(6): p. 1330-1336.

32. Colloca, G.A., A. Venturino, and D. Guarneri, Different variables predict the outcome of patients with synchronous versus metachronous metastases of colorectal cancer. Clin Transl Oncol, 2020.

33. Memeo, R., et al., Postoperative Infectious Complications Impact Long-Term Survival in Patients Who Underwent Hepatectomies for Colorectal Liver Metastases: a Propensity Score Matching Analysis. J Gastrointest Surg, 2018.

34. Dupre, A., et al., Curative-intent treatment of recurrent colorectal liver metastases: $A$ comparison between ablation and resection. Eur J Surg Oncol, 2017. 43(10): p. 1901-1907.

35. Imai, K., et al., Impact of Surgical Treatment for Recurrence After 2-Stage Hepatectomy for Colorectal Liver Metastases, on Patient Outcome. Ann Surg, 2017.

36. Hanham, I.W., K.A. Newton, and G. Westbury, Seventy-five cases of solid tumours treated by a modified quadruple chemotherapy regime. Br J Cancer, 1971. 25(3): p. 462-78.

37. Yoon, S.S. and K.K. Tanabe, Multidisciplinary management of metastatic colorectal cancer. Surgical oncology, 1998. 7(3-4): p. 197-207.

38. Levi, F., et al., Oxaliplatin activity against metastatic colorectal cancer. A phase II study of 5day continuous venous infusion at circadian rhythm modulated rate. European journal of cancer (Oxford, England : 1990), 1993. 29A(9): p. 1280-1284. 
39. Beppu, T., et al., Chemotherapy and targeted therapy for patients with initially unresectable colorectal liver metastases, focusing on conversion hepatectomy and long-term survival. Ann Surg Oncol, 2014. 21 Suppl 3: p. S405-13.

40. Jones, R.P. and G.J. Poston, Resection of Liver Metastases in Colorectal Cancer in the Era of Expanding Systemic Therapy. Annu Rev Med, 2017. 68: p. 183-196.

41. Nordlinger, B., et al., Perioperative chemotherapy with FOLFOX4 and surgery versus surgery alone for resectable liver metastases from colorectal cancer (EORTC Intergroup trial 40983): a randomised controlled trial. Lancet, 2008. 371(9617): p. 1007-16.

42. Nordlinger, B., et al., Perioperative FOLFOX4 chemotherapy and surgery versus surgery alone for resectable liver metastases from colorectal cancer (EORTC 40983): long-term results of a randomised, controlled, phase 3 trial. The Lancet. Oncology, 2013. 14(12): p. 1208-1215.

43. Adam, R., et al., Is perioperative chemotherapy useful for solitary, metachronous, colorectal liver metastases? Ann Surg, 2010. 252(5): p. 774-87.

44. Tanaka, K., et al., Role of neoadjuvant chemotherapy in the treatment of multiple colorectal metastases to the liver. Br J Surg, 2003. 90(8): p. 963-9.

45. Adam, R., et al., The oncosurgery approach to managing liver metastases from colorectal cancer: a multidisciplinary international consensus. Oncologist, 2012. 17(10): p. 1225-39.

46. Adam, R., et al., Rescue surgery for unresectable colorectal liver metastases downstaged by chemotherapy: a model to predict long-term survival. Ann Surg, 2004. 240(4): p. 644-57; discussion 657-8.

47. Maeda, Y., et al., Long-Term Outcomes of Conversion Hepatectomy for Initially Unresectable Colorectal Liver Metastases. Ann Surg Oncol, 2016. 23 Suppl 2: p. S242-8.

48. Capussotti, L., et al., Neoadjuvant chemotherapy and resection for initially irresectable colorectal liver metastases. Br J Surg, 2006. 93(8): p. 1001-6.

49. Kawamura, J., et al., Clinical efficacy of liver resection after downsizing systemic chemotherapy for initially unresectable liver metastases. World journal of surgical oncology, 2016. 14: p. 56-56.

50. Nuzzo, G., et al., Liver resection for primarily unresectable colorectal metastases downsized by chemotherapy. J Gastrointest Surg, 2007. 11(3): p. 318-24.

51. Ecker, D.M., S.D. Jones, and H.L. Levine, The therapeutic monoclonal antibody market. mAbs, 2015. 7(1): p. 9-14.

52. Rosen, L.S., I.A. Jacobs, and R.L. Burkes, Bevacizumab in Colorectal Cancer: Current Role in Treatment and the Potential of Biosimilars. Targeted oncology, 2017. 12(5): p. 599-610.

53. Tomasello, G., et al., FOLFOXIRI Plus Bevacizumab as Conversion Therapy for Patients With Initially Unresectable Metastatic Colorectal Cancer: A Systematic Review and Pooled Analysis. JAMA oncology, 2017. 3(7): p. e170278-e170278.

54. Petrelli, F., et al., Different Toxicity of Cetuximab and Panitumumab in Metastatic Colorectal Cancer Treatment: A Systematic Review and Meta-Analysis. Oncology, 2018. 94(4): p. 191199.

55. Fornasier, G., S. Francescon, and P. Baldo, An Update of Efficacy and Safety of Cetuximab in Metastatic Colorectal Cancer: A Narrative Review. Advances in therapy, 2018. 35(10): p. 1497-1509.

56. Folprecht, G., et al., Tumour response and secondary resectability of colorectal liver metastases following neoadjuvant chemotherapy with cetuximab: the CELIM randomised phase 2 trial. The Lancet. Oncology, 2010. 11(1): p. 38-47.

57. Carrato, A., et al., First-line panitumumab plus FOLFOX4 or FOLFIRI in colorectal cancer with multiple or unresectable liver metastases: A randomised, phase II trial (PLANET-TTD).

European journal of cancer (Oxford, England : 1990), 2017. 81: p. 191-202.

58. Fukuoka, K., et al., Hepatectomy for Colorectal Cancer Liver Metastases in the Era of Modern Preoperative Chemotherapy: Evaluation of Postoperative Complications. World journal of surgery, 2017. 41(4): p. 1073-1081. 
59. Ribeiro, H.S.C., et al., Extended preoperative chemotherapy, extent of liver resection and blood transfusion are predictive factors of liver failure following resection of colorectal liver metastasis. European journal of surgical oncology : the journal of the European Society of Surgical Oncology and the British Association of Surgical Oncology, 2013. 39(4): p. 380-385.

60. Shindoh, J., et al., Optimal future liver remnant in patients treated with extensive preoperative chemotherapy for colorectal liver metastases. Ann Surg Oncol, 2013. 20(8): p. 2493-500.

61. Dede, K., et al., Bevacizumab treatment before resection of colorectal liver metastases: safety, recovery of liver function, pathologic assessment. Pathology oncology research : POR, 2013. 19(3): p. 501-508.

62. Neeff, H.P., et al., Impact of preoperative targeted therapy on postoperative complications after resection of colorectal liver metastases. International journal of colorectal disease, 2012. 27(5): p. 635-645.

63. Rubbia-Brandt, L., et al., Severe hepatic sinusoidal obstruction associated with oxaliplatinbased chemotherapy in patients with metastatic colorectal cancer. Annals of oncology : official journal of the European Society for Medical Oncology, 2004. 15(3): p. 460-466.

64. Khan, A.Z., G. Morris-Stiff, and M. Makuuchi, Patterns of chemotherapy-induced hepatic injury and their implications for patients undergoing liver resection for colorectal liver metastases. Journal of hepato-biliary-pancreatic surgery, 2009. 16(2): p. 137-144.

65. Zhao, J., et al., Systematic review of the influence of chemotherapy-associated liver injury on outcome after partial hepatectomy for colorectal liver metastases. The British journal of surgery, 2017. 104(8): p. 990-1002.

66. Aussilhou, B., et al., Preoperative liver hypertrophy induced by portal flow occlusion before major hepatic resection for colorectal metastases can be impaired by bevacizumab. Ann Surg Oncol, 2009. 16(6): p. 1553-9.

67. Sturesson, C., I. Keussen, and K.G. Tranberg, Prolonged chemotherapy impairs liver regeneration after portal vein occlusion - an audit of 26 patients. Eur J Surg Oncol, 2010. 36(4): p. 358-64.

68. Goere, D., et al., Chemotherapy does not impair hypertrophy of the left liver after right portal vein obstruction. J Gastrointest Surg, 2006. 10(3): p. 365-70.

69. Tanaka, K., et al., Influence of chemotherapy on liver regeneration induced by portal vein embolization or first hepatectomy of a staged procedure for colorectal liver metastases. J Gastrointest Surg, 2010. 14(2): p. 359-68.

70. C, L., Ein Fall von Resecktion eines linksseitigen Schnurlappens der Leber. Berl Klin Wochenschr, 1888. 1888;25:37.

71. Keen, W.W., IV. Report of a Case of Resection of the Liver for the Removal of a Neoplasm, with a Table of Seventy-six Cases of Resection of the Liver for Hepatic Tumors. Ann Surg, 1899. 30(3): p. 267-83.

72. Couinaud, C., [Liver lobes and segments: notes on the anatomical architecture and surgery of the liver ]. Presse Med, 1954. 62(33): p. 709-12.

73. Kurtz, A.B., C.S. Rubin, and B.B. Goldberg, Ultrasound and computed tomography of the liver. Crit Rev Diagn Imaging, 1982. 18(4): p. 279-317.

74. Makuuchi, M., H. Hasegawa, and S. Yamazaki, Intraoperative ultrasonic examination for hepatectomy. Ultrasound Med Biol, 1983. Suppl 2: p. 493-7.

75. Bismuth, H., Surgical anatomy and anatomical surgery of the liver. World J Surg, 1982. 6(1): p. 3-9.

76. Pamecha, V., et al., Techniques for liver parenchymal transection: a meta-analysis of randomized controlled trials. HPB (Oxford), 2009. 11(4): p. 275-81.

77. Poon, R.T.P., Current techniques of liver transection. HPB : the official journal of the International Hepato Pancreato Biliary Association, 2007. 9(3): p. 166-173. 
78. Heriot, A.G. and N.D. Karanjia, A review of techniques for liver resection. Ann R Coll Surg Engl, 2002. 84(6): p. 371-80.

79. Pringle, J.H., V. Notes on the Arrest of Hepatic Hemorrhage Due to Trauma. Ann Surg, 1908. 48(4): p. 541-9.

80. Zhang, X.L., et al., Effectiveness and safety of controlled venous pressure in liver surgery: $a$ systematic review and network meta-analysis. Biomed Res Int, 2015. 2015: p. 290234.

81. Strasberg, S.M., Nomenclature of hepatic anatomy and resections: a review of the Brisbane 2000 system. J Hepatobiliary Pancreat Surg, 2005. 12(5): p. 351-5.

82. Cedermark, B.J., et al., The value of liver scan in the follow-up study of patients with adenocarcinoma of the colon and rectum. Surg Gynecol Obstet, 1977. 144(5): p. 745-8.

83. Lamb, G. and I. Taylor, An assessment of ultrasound scanning in the recognition of colorectal liver metastases. Ann R Coll Surg Engl, 1982. 64(6): p. 391-3.

84. Renzulli, M., et al., Imaging of Colorectal Liver Metastases: New Developments and Pending Issues. Cancers (Basel), 2020. 12(1).

85. Bipat, S., et al., Colorectal liver metastases: CT, MR imaging, and PET for diagnosis--metaanalysis. Radiology, 2005. 237(1): p. 123-31.

86. Floriani, l., et al., Performance of imaging modalities in diagnosis of liver metastases from colorectal cancer: a systematic review and meta-analysis. J Magn Reson Imaging, 2010. 31(1): p. 19-31.

87. Niekel, M.C., S. Bipat, and J. Stoker, Diagnostic imaging of colorectal liver metastases with CT, MR imaging, FDG PET, and/or FDG PET/CT: a meta-analysis of prospective studies including patients who have not previously undergone treatment. Radiology, 2010. 257(3): p. 674-84.

88. Vilgrain, V., et al., A meta-analysis of diffusion-weighted and gadoxetic acid-enhanced MR imaging for the detection of liver metastases. Eur Radiol, 2016. 26(12): p. 4595-4615.

89. Choi, S.H., et al., Diagnostic performance of CT, gadoxetate disodium-enhanced MRI, and $P E T / C T$ for the diagnosis of colorectal liver metastasis: Systematic review and meta-analysis. J Magn Reson Imaging, 2018. 47(5): p. 1237-1250.

90. Zhang, L., et al., Detection of liver metastases on gadobenate dimeglumine-enhanced MRI: systematic review, meta-analysis, and similarities with gadoxetate-enhanced MRI. Eur Radiol, 2019. 29(10): p. 5205-5216.

91. Langella, S., et al., Intraoperative Ultrasound Staging for Colorectal Liver Metastases in the Era of Liver-Specific Magnetic Resonance Imaging: Is It Still Worthwhile? J Oncol, 2019. 2019: p. 1369274.

92. Granata, V., et al., Liver radiologic findings of chemotherapy-induced toxicity in liver colorectal metastases patients. Eur Rev Med Pharmacol Sci, 2019. 23(22): p. 9697-9706.

93. van Kessel, C.S., et al., Preoperative imaging of colorectal liver metastases after neoadjuvant chemotherapy: a meta-analysis. Ann Surg Oncol, 2012. 19(9): p. 2805-13.

94. Gallinger, S., et al., Liver resection for colorectal cancer metastases. Curr Oncol, 2013. 20(3): p. e255-65.

95. Shi, J.-H. and P.-D. Line, Hallmarks of postoperative liver regeneration: An updated insight on the regulatory mechanisms. Journal of gastroenterology and hepatology, 2019: $\mathrm{p}$. 10.1111/jgh.14944.

96. Michalopoulos, G.K., Advances in liver regeneration. Expert Rev Gastroenterol Hepatol, 2014. 8(8): p. 897-907.

97. García-Pérez, R., et al., Associated Liver Partition and Portal Vein Ligation (ALPPS) vs Selective Portal Vein Ligation (PVL) for Staged Hepatectomy in a Rat Model. Similar Regenerative Response? PloS one, 2015. 10(12): p. e0144096-e0144096.

98. Sheng, R.-F., et al., Assessment of liver regeneration after associating liver partition and portal vein ligation for staged hepatectomy: a comparative study with portal vein ligation. 
HPB : the official journal of the International Hepato Pancreato Biliary Association, 2018. 20(4): p. 305-312.

99. Borger, P., et al., Exploration of the Transcriptional Landscape of ALPPS Reveals the Pathways of Accelerated Liver Regeneration. Frontiers in oncology, 2019. 9: p. 1206-1206.

100. Cieslak, K.P., et al., Liver function declines with increased age. HPB : the official journal of the International Hepato Pancreato Biliary Association, 2016. 18(8): p. 691-696.

101. Fernandes, A.I., et al., Functional hepatocellular regeneration in elderly patients undergoing hepatectomy. Liver Int, 2015. 35(4): p. 1116-23.

102. Amini, N., et al., Liver regeneration after major liver hepatectomy: Impact of body mass index. Surgery, 2016. 160(1): p. 81-91.

103. Truant, S., et al., Volumetric gain of the liver after major hepatectomy in obese patients: $a$ case-matched study in 84 patients. Annals of surgery, 2013. 258(5): p. 696-704.

104. Schulze-Hagen, M., et al., Correlation Between Sarcopenia and Growth Rate of the Future Liver Remnant After Portal Vein Embolization in Patients with Colorectal Liver Metastases. Cardiovascular and interventional radiology, 2020: p. 10.1007/s00270-020-02416-6.

105. Denbo, J.W., et al., Overall Body Composition and Sarcopenia Are Associated with Poor Liver Hypertrophy Following Portal Vein Embolization. Journal of gastrointestinal surgery : official journal of the Society for Surgery of the Alimentary Tract, 2020: p. 10.1007/s11605-02004522-9.

106. Rahbari, N.N., et al., Posthepatectomy liver failure: a definition and grading by the International Study Group of Liver Surgery (ISGLS). Surgery, 2011. 149(5): p. 713-724.

107. Balzan, S., et al., The "50-50 criteria" on postoperative day 5: an accurate predictor of liver failure and death after hepatectomy. Annals of surgery, 2005. 242(6): p. 824-829.

108. Kishi, Y., et al., Three hundred and one consecutive extended right hepatectomies: evaluation of outcome based on systematic liver volumetry. Annals of surgery, 2009. 250(4): p. 540-548.

109. Abdalla, E.K., et al., Extended hepatectomy in patients with hepatobiliary malignancies with and without preoperative portal vein embolization. Arch Surg, 2002. 137(6): p. 675-80; discussion 680-1.

110. Narita, M., et al., What is a safe future liver remnant size in patients undergoing major hepatectomy for colorectal liver metastases and treated by intensive preoperative chemotherapy? Ann Surg Oncol, 2012. 19(8): p. 2526-38.

111. Imamura, H., et al., Preoperative portal vein embolization: an audit of 84 patients. Hepatology, 1999. 29(4): p. 1099-105.

112. Vauthey, J.N., et al., Standardized measurement of the future liver remnant prior to extended liver resection: methodology and clinical associations. Surgery, 2000. 127(5): p. 512-519.

113. Ribero, D., Y.S. Chun, and J.-N. Vauthey, Standardized liver volumetry for portal vein embolization. Seminars in interventional radiology, 2008. 25(2): p. 104-109.

114. Vauthey, J.N., et al., Body surface area and body weight predict total liver volume in Western adults. Liver Transpl, 2002. 8(3): p. 233-40.

115. Martel, G., et al., Comparison of techniques for volumetric analysis of the future liver remnant: implications for major hepatic resections. HPB (Oxford), 2015. 17(12): p. 1051-7.

116. Guglielmi, A., et al., How much remnant is enough in liver resection? Dig Surg, 2012. 29(1): p. 6-17.

117. Shindoh, J., et al., Kinetic growth rate after portal vein embolization predicts posthepatectomy outcomes: toward zero liver-related mortality in patients with colorectal liver metastases and small future liver remnant. J Am Coll Surg, 2013. 216(2): p. 201-9.

118. Adam, R., et al., Two-stage hepatectomy approach for initially unresectable colorectal hepatic metastases. Surg Oncol Clin N Am, 2007. 16(3): p. 525-36, viii.

119. Bismuth, $\mathrm{H}_{\text {., }}$ et al., Resection of nonresectable liver metastases from colorectal cancer after neoadjuvant chemotherapy. Ann Surg, 1996. 224(4): p. 509-20; discussion 520-2. 
120. Tsai, S., et al., Two-stage strategy for patients with extensive bilateral colorectal liver metastases. HPB (Oxford), 2010. 12(4): p. 262-9.

121. Vigano, L., et al., Drop-out between the two liver resections of two-stage hepatectomy. Patient selection or loss of chance? Eur J Surg Oncol, 2016.

122. Regimbeau, J.M., et al., Feasibility, safety and efficacy of two-stage hepatectomy for bilobar liver metastases of colorectal cancer: a LiverMetSurvey analysis. HPB (Oxford), 2017. 19(5): p. 396-405.

123. Lam, V.W., et al., A systematic review of two-stage hepatectomy in patients with initially unresectable colorectal liver metastases. HPB (Oxford), 2013. 15(7): p. 483-91.

124. Makuuchi, M., et al., Preoperative portal embolization to increase safety of major hepatectomy for hilar bile duct carcinoma: a preliminary report. Surgery, 1990. 107(5): p. 521-7.

125. Biggemann, L., et al., Future liver remnant growth after various portal vein embolization regimens: a quantitative comparison. Minimally invasive therapy \& allied technologies: MITAT : official journal of the Society for Minimally Invasive Therapy, 2019: p. 1-9.

126. Dhaliwal, S.K., et al., Portal Vein Embolization: Correlation of Future Liver Remnant Hypertrophy to Type of Embolic Agent Used. Canadian Association of Radiologists journal = Journal I'Association canadienne des radiologistes, 2018. 69(3): p. 316-321.

127. Jaberi, A., et al., Comparison of Clinical Outcomes following Glue versus Polyvinyl Alcohol Portal Vein Embolization for Hypertrophy of the Future Liver Remnant prior to Right Hepatectomy. J Vasc Interv Radiol, 2016. 27(12): p. 1897-1905.e1.

128. Guiu, B., et al., Portal vein embolization before right hepatectomy: improved results using $n$ butyl-cyanoacrylate compared to microparticles plus coils. Cardiovasc Intervent Radiol, 2013. 36(5): p. 1306-12.

129. Soyer, P., et al., Hepatic metastases from colorectal cancer: influence of hepatic volumetric analysis on surgical decision making. Radiology, 1992. 184(3): p. 695-697.

130. Abdalla, E.K., et al., Improving resectability of hepatic colorectal metastases: expert consensus statement. Ann Surg Oncol, 2006. 13(10): p. 1271-80.

131. Azoulay, D., et al., Resection of nonresectable liver metastases from colorectal cancer after percutaneous portal vein embolization. Annals of surgery, 2000. 231(4): p. 480-486.

132. Jaeck, D., et al., A two-stage hepatectomy procedure combined with portal vein embolization to achieve curative resection for initially unresectable multiple and bilobar colorectal liver metastases. Ann Surg, 2004. 240(6): p. 1037-49; discussion 1049-51.

133. Shindoh, J., et al., Safety and efficacy of portal vein embolization before planned major or extended hepatectomy: an institutional experience of 358 patients. J Gastrointest Surg, 2014. 18(1): p. 45-51.

134. Huang, S.Y., et al., Efficacy and safety of portal vein embolization for two-stage hepatectomy in patients with colorectal liver metastasis. J Vasc Interv Radiol, 2014. 25(4): p. 608-17.

135. Ardito, F., et al., Right and extended-right hepatectomies for unilobar colorectal metastases: impact of portal vein embolization on long-term outcome and liver recurrence. Surgery, 2013. 153(6): p. 801-10.

136. Simoneau, E., et al., Portal vein embolization and its effect on tumour progression for colorectal cancer liver metastases. Br J Surg, 2015. 102(10): p. 1240-9.

137. Fischer, C., et al., Chemotherapy after portal vein embolization to protect against tumor growth during liver hypertrophy before hepatectomy. JAMA Surg, 2013. 148(12): p. 1103-8.

138. de Graaf, W., et al., Increase in future remnant liver function after preoperative portal vein embolization. Br J Surg, 2011. 98(6): p. 825-34.

139. Collin, Y., et al., Portal vein embolization does not affect the long-term survival and risk of cancer recurrence among colorectal liver metastases patients: A prospective cohort study. Int J Surg, 2019. 61: p. 42-47. 
140. Huiskens, J., et al., Does portal vein embolization prior to liver resection influence the oncological outcomes - A propensity score matched comparison. Eur J Surg Oncol, 2018. 44(1): p. 108-114.

141. Rous, P. and L.D. Larimore, Relation of the Portal Blood to Liver Maintenance : A Demonstration of Liver Atrophy Conditional on Compensation. J Exp Med, 1920. 31(5): p. 609-32.

142. Kameoka, N., et al., Advantage of preoperative portal vein occlusion for hepatectomy that exceeds portal vein occluded lobes. Surgery, 1998. 123(5): p. 545-53.

143. Kianmanesh, R., et al., Right portal vein ligation: a new planned two-step all-surgical approach for complete resection of primary gastrointestinal tumors with multiple bilateral liver metastases. J Am Coll Surg, 2003. 197(1): p. 164-70.

144. Broering, D.C., et al., Portal vein embolization vs. portal vein ligation for induction of hypertrophy of the future liver remnant. J Gastrointest Surg, 2002. 6(6): p. 905-13; discussion 913.

145. Denys, A.L., et al., Failure of right portal vein ligation to induce left lobe hypertrophy due to intrahepatic portoportal collaterals: successful treatment with portal vein embolization. AJR Am J Roentgenol, 1999. 173(3): p. 633-5.

146. Aussilhou, B., et al., Right portal vein ligation is as efficient as portal vein embolization to induce hypertrophy of the left liver remnant. J Gastrointest Surg, 2008. 12(2): p. 297-303.

147. Shindoh, J., et al., Analysis of the efficacy of portal vein embolization for patients with extensive liver malignancy and very low future liver remnant volume, including a comparison with the associating liver partition with portal vein ligation for staged hepatectomy approach. J Am Coll Surg, 2013. 217(1): p. 126-33; discussion 133-4.

148. Schnitzbauer, A.A., et al., Right portal vein ligation combined with in situ splitting induces rapid left lateral liver lobe hypertrophy enabling 2-staged extended right hepatic resection in small-for-size settings. Ann Surg, 2012. 255(3): p. 405-14.

149. Nadalin, S., et al., Indications and limits for associating liver partition and portal vein ligation for staged hepatectomy (ALPPS). Lessons Learned from 15 cases at a single centre. $Z$ Gastroenterol, 2014. 52(1): p. 35-42.

150. Sala, S., et al., Our initial experience with ALPPS technique: encouraging results. Updates Surg, 2012. 64(3): p. 167-72.

151. Alvarez, F.A., et al., Associating liver partition and portal vein ligation for staged hepatectomy (ALPPS): tips and tricks. J Gastrointest Surg, 2013. 17(4): p. 814-21.

152. Li, J., et al., ALPPS in right trisectionectomy: a safe procedure to avoid postoperative liver failure? Journal of gastrointestinal surgery : official journal of the Society for Surgery of the Alimentary Tract, 2013. 17(5): p. 956-961.

153. de Santibanes, E., F.A. Alvarez, and V. Ardiles, How to avoid postoperative liver failure: $a$ novel method. World J Surg, 2012. 36(1): p. 125-8.

154. Petrowsky, H., et al., Is partial-ALPPS safer than ALPPS? A single-center experience. Annals of surgery, 2015. 261(4): p. e90-e92.

155. Robles, R., et al., Tourniquet modification of the associating liver partition and portal ligation for staged hepatectomy procedure. The British journal of surgery, 2014. 101(9): p. 11291134.

156. Machado, M.A., et al., Totally laparoscopic ALPPS for multiple and bilobar colorectal metastases (with video). Journal of visceral surgery, 2017. 154(2): p. 131-132.

157. Cillo, U., et al., Totally Laparoscopic Microwave Ablation and Portal Vein Ligation for Staged Hepatectomy : A New Minimally Invasive Two-Stage Hepatectomy. Annals of surgical oncology, 2015. 22(8): p. 2787-2788.

158. Gall, T.M.H., et al., Radio-frequency-assisted Liver Partition with Portal vein ligation (RALPP) for liver regeneration. Annals of surgery, 2015. 261(2): p. e45-e46. 
159. Vicente, E., et al., First ALPPS procedure using a total robotic approach. Surgical oncology, 2016. 25(4): p. 457-457.

160. Sparrelid, E., et al., Rescue ALPPS is efficient and safe after failed portal vein occlusion in patients with colorectal liver metastases. Langenbecks Arch Surg, 2017. 402(1): p. 69-75.

161. Tschuor, C., et al., Salvage parenchymal liver transection for patients with insufficient volume increase after portal vein occlusion -- an extension of the ALPPS approach. Eur J Surg Oncol, 2013. 39(11): p. 1230-5.

162. Björnsson, B., T. Gasslander, and P. Sandström, In situ split of the liver when portal venous embolization fails to induce hypertrophy: a report of two cases. Case reports in surgery, 2013. 2013: p. 238675-238675.

163. Enne, M., et al., ALPPS as a salvage procedure after insufficient future liver remnant hypertrophy following portal vein occlusion. HPB (Oxford), 2017. 19(12): p. 1126-1129.

164. Schadde, E., et al., Monosegment ALPPS hepatectomy: extending resectability by rapid hypertrophy. Surgery, 2015. 157(4): p. 676-689.

165. Lang, S.A., et al., Long-term results after in-situ split (ISS) liver resection. Langenbeck's archives of surgery, 2015. 400(3): p. 361-369.

166. Schadde, E., et al., Early survival and safety of ALPPS: first report of the International ALPPS Registry. Annals of surgery, 2014. 260(5): p. 829-838.

167. Linecker, M., et al., Risk Adjustment in ALPPS Is Associated With a Dramatic Decrease in Early Mortality and Morbidity. Ann Surg, 2017. 266(5): p. 779-786.

168. Truant, S., et al., Associating liver partition and portal vein ligation for staged hepatectomy (ALPPS): impact of the inter-stages course on morbi-mortality and implications for management. Eur J Surg Oncol, 2015. 41(5): p. 674-82.

169. Tanaka, K., et al., Associating liver partition and portal vein ligation for staged hepatectomy (ALPPS): short-term outcome, functional changes in the future liver remnant, and tumor growth activity. European journal of surgical oncology : the journal of the European Society of Surgical Oncology and the British Association of Surgical Oncology, 2015. 41(4): p. 506512.

170. Sparrelid, E., et al., Dynamic Evaluation of Liver Volume and Function in Associating Liver Partition and Portal Vein Ligation for Staged Hepatectomy. J Gastrointest Surg, 2017. 21(6): p. 967-974.

171. Cieslak, K.P., et al., Assessment of Liver Function Using (99m)Tc-Mebrofenin Hepatobiliary Scintigraphy in ALPPS (Associating Liver Partition and Portal Vein Ligation for Staged Hepatectomy). Case reports in gastroenterology, 2015. 9(3): p. 353-360.

172. Truant, S., et al., Drop of Total Liver Function in the Interstages of the New Associating Liver Partition and Portal Vein Ligation for Staged Hepatectomy Technique: Analysis of the "Auxiliary Liver" by HIDA Scintigraphy. Annals of surgery, 2016. 263(3): p. e33-e34.

173. Ratti, F., et al., Strategies to Increase the Resectability of Patients with Colorectal Liver Metastases: A Multi-center Case-Match Analysis of ALPPS and Conventional Two-Stage Hepatectomy. Ann Surg Oncol, 2015. 22(6): p. 1933-42.

174. Adam, R., et al., Outcome after associating liver partition and portal vein ligation for staged hepatectomy and conventional two-stage hepatectomy for colorectal liver metastases. $\mathrm{Br} \mathrm{J}$ Surg, 2016. 103(11): p. 1521-9.

175. Baumgart, J., et al., Two-Stage Hepatectomy and ALPPS for Advanced Bilateral Liver Metastases: a Tailored Approach Balancing Risk and Outcome. J Gastrointest Surg, 2019.

176. Schadde, E., et al., ALPPS offers a better chance of complete resection in patients with primarily unresectable liver tumors compared with conventional-staged hepatectomies: results of a multicenter analysis. World J Surg, 2014. 38(6): p. 1510-9.

177. Olthof, P.B., et al., Survival after associating liver partition and portal vein ligation for staged hepatectomy (ALPPS) for advanced colorectal liver metastases: A case-matched comparison with palliative systemic therapy. Surgery, 2017. 161(4): p. 909-919. 
178. Sandstrom, P., et al., ALPPS Improves Resectability Compared With Conventional Two-stage Hepatectomy in Patients With Advanced Colorectal Liver Metastasis: Results From a Scandinavian Multicenter Randomized Controlled Trial (LIGRO Trial). Ann Surg, 2018. 267(5): p. 833-840.

179. Torzilli, G., et al., Outcomes of enhanced one-stage ultrasound-guided hepatectomy for bilobar colorectal liver metastases compared to those of ALPPS: a multicenter case-match analysis. HPB (Oxford), 2019.

180. Jiao, L.R., et al., Rapid Induction of Liver Regeneration for Major Hepatectomy (REBIRTH): A Randomized Controlled Trial of Portal Vein Embolisation versus ALPPS Assisted with Radiofrequency. Cancers (Basel), 2019. 11(3).

181. Arrow, K.J., Uncertainty and the welfare economics of medical care. 1963. Bulletin of the World Health Organization, 2004. 82(2): p. 141-149.

182. K, M., Capital. 1887, London: Swan Sonnenschein.

183. Ferraz-Nunes J, K.I., Hälsoekonomi - begrepp och tillämpningar. Second edition ed. 2012: Studentlitteratur. 297.

184. Drummond, M., Methods for the Economic Evaluation of Health Care Programmes. 2015.

185. Ottolini, F.L., et al., The complex interface between economy and healthcare: An introductory overview for clinicians. Eur J Intern Med, 2016. 36: p. 1-6.

186. EuroQol--a new facility for the measurement of health-related quality of life. Health Policy, 1990. 16(3): p. 199-208.

187. Brooks, R., EuroQol: the current state of play. Health Policy, 1996. 37(1): p. 53-72.

188. Hälsoekonomiska Utvärderingar, S.b.f.m.u. (SBU), Editor. 2017.

189. Langenhoff, B.S., et al., Quality of life after surgical treatment of colorectal liver metastases. Br J Surg, 2006. 93(8): p. 1007-14.

190. Wanis, K.N., et al., Intermediate-term survival and quality of life outcomes in patients with advanced colorectal liver metastases undergoing associating liver partition and portal vein ligation for staged hepatectomy. Surgery, 2018. 163(4): p. 691-697.

191. Roberts, K.J., et al., Cost-utility analysis of operative versus non-operative treatment for colorectal liver metastases. Br J Surg, 2015. 102(4): p. 388-98.

192. Gazelle, G.S., et al., Cost-effectiveness of hepatic metastasectomy in patients with metastatic colorectal carcinoma: a state-transition Monte Carlo decision analysis. Ann Surg, 2003. 237(4): p. 544-55.

193. Beard, S.M., et al., Hepatic resection for colorectal liver metastases: A cost-effectiveness analysis. Ann Surg, 2000. 232(6): p. 763-76.

194. Johannesson, M. and M.C. Weinstein, On the decision rules of cost-effectiveness analysis. J Health Econ, 1993. 12(4): p. 459-67.

195. Dolan, P., Modeling valuations for EuroQol health states. Med Care, 1997. 35(11): p. 1095108.

196. Kremer, M., et al., Impact of Neoadjuvant Chemotherapy on Hypertrophy of the Future Liver Remnant after Associating Liver Partition and Portal Vein Ligation for Staged Hepatectomy. J Am Coll Surg, 2015. 221(3): p. 717-728.e1.

197. Sturesson, C., et al., Limiting factors for liver regeneration after a major hepatic resection for colorectal cancer metastases. HPB (Oxford), 2013. 15(8): p. 646-52.

198. Dello, S.A., et al., Influence of preoperative chemotherapy on CT volumetric liver regeneration following right hemihepatectomy. World J Surg, 2014. 38(2): p. 497-504.

199. Millet, G., et al., Volumetric analysis of remnant liver regeneration after major hepatectomy in bevacizumab-treated patients: a case-matched study in 82 patients. Ann Surg, 2012.

256(5): p. 755-61; discussion 761-2.

200. Robinson, S.M., et al., Pathogenesis of FOLFOX induced sinusoidal obstruction syndrome in a murine chemotherapy model. J Hepatol, 2013. 59(2): p. 318-26. 
201. Björnsson, B., et al., Segment 4 occlusion in portal vein embolization increase future liver remnant hypertrophy - A Scandinavian cohort study. Int J Surg, 2020. 75: p. 60-65.

202. Kambakamba, P., et al., Short Chemotherapy-Free Interval Improves Oncological Outcome in Patients Undergoing Two-Stage Hepatectomy for Colorectal Liver Metastases. Ann Surg Oncol, 2016. 23(12): p. 3915-3923.

203. Aloia, T.A. and J.N. Vauthey, Associating liver partition and portal vein ligation for staged hepatectomy (ALPPS): what is gained and what is lost? Ann Surg, 2012. 256(3): p. e9; author reply e16-9.

204. Hwang, S., et al., Preoperative Sequential Portal and Hepatic Vein Embolization in Patients with Hepatobiliary Malignancy. World J Surg, 2015. 39(12): p. 2990-8.

205. Panaro, F., et al., Perioperative impact of liver venous deprivation compared with portal venous embolization in patients undergoing right hepatectomy: preliminary results from the pioneer center. Hepatobiliary Surg Nutr, 2019. 8(4): p. 329-337.

206. Guiu, B., et al., Simultaneous trans-hepatic portal and hepatic vein embolization before major hepatectomy: the liver venous deprivation technique. Eur Radiol, 2016. 26(12): p. 4259-4267.

207. Guiu, B., et al., Extended liver venous deprivation before major hepatectomy induces marked and very rapid increase in future liver remnant function. Eur Radiol, 2017. 27(8): p. 33433352.

208. Fretland Å, A., et al., Laparoscopic Versus Open Resection for Colorectal Liver Metastases: The OSLO-COMET Randomized Controlled Trial. Ann Surg, 2018. 267(2): p. 199-207. 



\section{Papers}

The papers associated with this thesis have been removed for copyright reasons. For more details about these see:

http://urn.kb.se/resolve?urn=urn:nbn:se:liu:diva-165372 


\section{FACULTY OF MEDICINE AND HEALTH SCIENCES}

Linköping University Medical Dissertation No. 1738, 2020

Department of BKV

Linköping University

SE-581 83 Linköping, Sweden

www.liu.se 